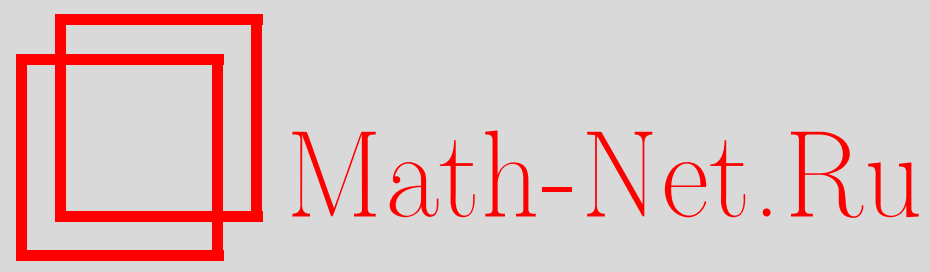

С. А. Кулешов, О многообразии модулей стабильных расслоений на квадрике, Матем. заметки, 1997, том 62, выпуск $6,843-864$

DOI: https://doi.org/10.4213/mzm1674

Использование Общероссийского математического портала Math-Net.Ru подразумевает, что вы прочитали и согласны с пользовательским соглашением http://www . mathnet.ru/rus/agreement

Параметры загрузки:

IP : 18.207 .199 .55

26 апреля 2023 г., 12:54:31 


\section{О МНОГООБРАЗИИ МОДУЛЕЙ СТАБИЛЬНЫХ РАССЛОЕНИЙ НА КВАДРИКЕ}

\section{С. А. Кулешов}

В статье исследуется связь стабильных представлений колчанов и стабильных пучков. На основе этой связи строятся тонкие гладкие компактные многообразия модулей стабильных пучков на квадрике.

Библиограффия: 9 названий.

Введение. Цель данной статьи - доказательство существования и явное описание серии многообразий модулей $\mathscr{M}_{k}=\mathscr{M}\left(r, c_{1}, k\right)$ стабильных пучков $E$ на гладкой проективной двумерной квадрике $Q$ ранга $r$ с первым классом Черна $c_{1}$ и вторьм классом Черна $c_{2}=k$. Причем многообразия должны удовлетворять следующим требованиям:

1) все многообразия $\mathscr{M}_{k}$ тонкие гладкие и компактные;

2 ) на каждом многообразии $\mathscr{M}_{k}$ существует расслоение $\mathscr{H}$, слой которого над точкой $[E]$, соответствующей пучку $E$, изоморфен $\mathrm{H}^{1}(E)$;

3) $\operatorname{dim} \mathscr{M}_{k}=n \cdot r(\mathscr{H})$ для некоторого натурального $n$.

Сформулированная задача связана с проверкой гипотезы $S$-двойственности, а именно: нужно проверить модулярные свойства производящей функции

$$
f(t)=\sum_{k \geqslant 0} a_{k} t^{k},
$$

где $a_{k}$ - старший класс Черна расслоения $n \mathscr{H}$ (прямая сумма $n$ слагаемых $\mathscr{H}$ ). Заметим, что ввиду требования 3) $a_{k}$ отождествляются с целыми числами.

Тонкость многообразия модулей $\mathscr{M}_{k}$ обеспечивает наличие универсального пучка $\mathscr{E}_{k}$ на прямом произведении $\mathscr{M}_{k} \times Q$, слой которого над $[E] \times Q$ изоморфен пучку $E$. Если $\pi_{\mathscr{M}}$ - проекция прямого произведения на первьй сомножитель, то $\mathscr{H}=R^{1}\left(\pi_{\mathscr{M}}\right)_{*}\left(\mathscr{E}_{k}\right)$ является искомым пучком. Локальная свобода пучка $\mathscr{H}$ обеспечивается условием $\mathrm{H}^{0}(E)=\mathrm{H}^{2}(E)=0$ для всех пучков из $\mathscr{M}_{k}$. Гладкость же многообразия $\mathscr{M}_{k}$ следует из того факта, что размерность касательного пространства к многообразию модулей стабильных пучков на поверхности Дель Пещц не зависит от точки.

Несложные вычисления, используюшие формулу Римана-Роха, показывают, что если стабильность понимать в смысле Мамфорда-Такемото относительно половины антиканонического класса квадрики - дивизора $(1,1)$ - и ограничиться рангом 3 (нужных пучков ранга 2 на квадрике нет), то подходящие первые классы Черна исчерпываются следуюшими: $(-7,-4),(-5,-5),(-2,1),(-1,-1)$. Коэффициент $n$ из требования 3$)$ при этом равен 6. 
Заметим, что 3-расслоения с такими дискретными инвариантами получаются друг из друга тензорным умножением на обратимые пучки и переходом к двойственному расслоению. Поэтому соответствующие многообразия бирационально изоморфны.

В процессе решения задачи выяснилось, что легче всего поддается описанию многообразие $\mathscr{M}(3,(-4,-1), k)$, которое, очевидно, изоморфно многообразиям

$$
\mathscr{M}(3,(-1,-1), k-2) \cong \mathscr{M}(3,(-7,-4), k+16)
$$

(универсальные пучки, соответствующие этим многообразиям, одинаковы с точностью до умножения на линейные расслоения, поднятые с квадрики). Поэтому в статье приведена конструкция многообразия $\mathscr{M}(3,(-4,-1), k)$. Более того, оказалось, что без дополнительных громоздких рассуждений получается следующая теорема.

ОСНОВНАЯ ТЕОРЕМА. Предположим, что на квадрике существует стабильный пучок $E$ ранга $r$ с $c_{1}=(a, b)$ и $c_{2}=k$, причем его дискретные инварианты удовлетворяют условиям:

1) $2 r+a+b=1$;

2) $-2 r<a+b<0$.

Тогда существует тонкое гладкое компактное многообразие модулей $\mathscr{M}(r,(a, b), k)$ таких пучков.

При ее доказательстве использовались методы построения многообразий модулей [1]-[3]. Схема рассуждений, использованная в статье, примерно следующая.

В п. 1 мы показьваем, что стабильные пучки на квадрике при некоторых необременительных условиях на дискретные инварианты являются когомологиями монад:

$$
0 \longrightarrow U_{0} \otimes \mathscr{O}(-1,-1) \stackrel{\alpha}{\longrightarrow} U_{1} \otimes \mathscr{O}(-1,0) \oplus U_{2} \otimes \mathscr{O}(0,-1) \stackrel{\beta}{\longrightarrow} U_{3} \otimes \mathscr{O} \longrightarrow 0 .
$$

По морфизму $\beta$ монады строится представление

$$
t_{\beta}: U_{1} \otimes H_{13}^{*} \oplus U_{2} \otimes H_{23}^{*} \rightarrow U_{3}
$$

колчана $Q_{21}$ (см. рис. 1,2 ).

В п. 2 мы приводим результаты А. Кинга о стабильных представлениях колчанов [4], необходимые для доказательства основной теоремы.

В п. 3 мы доказываем, что существует взаимно однозначное соответствие между стабильными представлениями $t_{\beta}$ и стабильными расслоениями $\operatorname{ker} \beta$, и строим тонкоегладкое компактное многообразие модулей стабильных расслоений, чьи дискретные инварианты удовлетворяют условиям:

i) $r(E)=r, c_{1}(E)=(a, b), c_{2}(E)=r+a+b+a b$;

ii) НОД $(r, a+b)=1,-2 r<a+b<0$.

Наконец, п. 4 посвящен доказательству основной теоремы. Кроме того, в конце мы доказьваем, что для любого натурального $k \geqslant 4$ существует стабильный пучок ранга 3 с $c_{1}=(-4,-1)$ и $c_{2}=k$, т.е. многообразие модулей $\mathscr{M}(3,(-4,-1), k)$ непусто.

Настоящая статья не столько решает конкретную задачу, сколько ставит новые. А именно:

1) вычислить старшие классы Черна $a_{k}$ пучка $6 \mathscr{H}$ на $\mathscr{M}_{k}$;

2) проверить модулярность функции (1);

3) обобщить основную теорему, используя другие полные исключительные наборы на квадрике. 
Автор признателен А. Н. Тюрину за постановку задачи, А. Л. Городенцеву за полезные обсуждения и А.И. Бондалу, указавшему на работу [4].

1. Монада на квадрике. Цель данного пункта - доказательство следующего утверждения.

ПРЕДЛОЖЕНИЕ 1. Любой пучок $X$ на квадрике $Q$, удовлетворяющий условиям

$$
\operatorname{Ext}^{q}(\mathscr{L}, X)=0 \quad \text { npu } q \neq 1, \quad \mathscr{L} \in(\mathscr{O}, \mathscr{O}(0,1), \mathscr{O}(1,0), \mathscr{O}(1,1)),
$$

является когомологией монады вида (2), в которой

$$
\begin{gathered}
U_{0}=\operatorname{Ext}^{1}(\mathscr{O}(1,1), X), \quad U_{1}=\operatorname{Ext}^{1}(\mathscr{O}(1,0), X), \\
U_{2}=\operatorname{Ext}^{1}(\mathscr{O}(0,1), X), \quad U_{3}=\operatorname{Ext}^{1}(\mathscr{O}, X) .
\end{gathered}
$$

ДокАЗАтЕЛЬСтво. Набор обратимых пучков $(\mathscr{O}(-1,-1), \mathscr{O}(-1,0), \mathscr{O}(0,-1), \mathscr{O})$ является полным исключительньм набором на квадрике, т.е.

минимальная полная триангулированная подкатегория в производной категории когерентных пучков на квадрике $D^{b}(Q)$, содержащая этот набор, совпадает со всей $D^{b}(Q)$ (будем обозначать ее через $\langle\mathscr{O}(-1,-1), \mathscr{O}(-1,0), \mathscr{O}(0,-1), \mathscr{O}\rangle_{\text {cat }}$ ); если переобозначить пучки набора как $\left(E_{0}, E_{1}, E_{2}, E_{3}\right)$, то

$$
\begin{gathered}
\operatorname{Ext}^{q}\left(E_{j}, E_{i}\right)=0 \text { при } j>i, q=0,1,2, \text { и при } i=j, q=1,2, \\
\operatorname{Ext}^{0}\left(E_{i}, E_{i}\right)=\mathbb{C}
\end{gathered}
$$

(см. [5]).

(Подробную информацию об исключительных наборах на квадрике и их перестройках можно найти в работах [6] и [2].)

Для доказательства предложения 1 нам потребуется одно замечание о полных исключительных наборах, приведенное в работе [5].

Пусть $\left(E_{0}, E_{1}, E_{2}, E_{3}\right)$ - полньй исключительный набор на квадрике и $X$ - некоторьй пучок, тогда

$X \in\left\langle E_{0}, \ldots, E_{s}\right\rangle_{\text {cat }}$, если и только если $\operatorname{Ext}^{q}\left(E_{j}, X\right)=0$ для всех $q$ и $j>s ;$

$X \in\left\langle E_{s}, \ldots, E_{3}\right\rangle_{\text {cat }}$, если и только если $\operatorname{Ext}^{q}\left(X, E_{j}\right)=0$ для всех $q$ и $j<s$.

Вернемся к доказательству предложения 1 и рассмотрим универсальное расширение

$$
0 \longrightarrow E \longrightarrow E^{\prime} \longrightarrow \mathrm{H}^{1}(E) \otimes \mathscr{O} \longrightarrow 0,
$$

т.е. расширение, заданное элементом

$$
e_{0} \in \operatorname{Ext}^{1}\left(H^{1}(E) \otimes \mathscr{O}, E\right) \cong \mathrm{H}^{1}(E)^{*} \otimes \mathrm{H}^{1}(E),
$$

соответствующим тождественному отображению. Из длинной точной когомологической последовательности, соответствуюшей универсальному расширению, следует, что пространства $\operatorname{Ext}^{q}\left(\mathscr{O}, E^{\prime}\right)$ тривиальны для всех $q$. Следовательно,

$$
E^{\prime} \in\langle\mathscr{O}(-1,-1), \mathscr{O}(-1,0), \mathscr{O}(0,-1)\rangle_{\text {cat }}
$$


Применяя к последовательности (4) функтор Ext $(\cdot, \mathscr{O}(-1,-1))$, мы получаем изоморфизмы

$$
\operatorname{Ext}^{q}(E, \mathscr{O}(-1,-1)) \cong \operatorname{Ext}^{q}\left(E^{\prime}, \mathscr{O}(-1,-1)\right) \quad \forall q .
$$

Следовательно, $\operatorname{Ext}^{q}\left(E^{\prime}, \mathscr{O}(-1,-1)\right)=0$ при $q \neq 1$. Используя предыдущий изоморфизм и теорему двойственности Серра, имеем

$$
\operatorname{Ext}^{1}\left(E^{\prime}, \mathscr{O}(-1,-1)\right)^{*} \cong \operatorname{Ext}^{1}(\mathscr{O}(1,1), E)
$$

Рассмотрим универсальное расширение

$$
0 \longrightarrow \operatorname{Ext}^{1}(\mathscr{O}(-1,-1), E) \otimes \mathscr{O}(-1,-1) \longrightarrow E^{\prime \prime} \longrightarrow E^{\prime} \longrightarrow 0
$$

Как и в предыдущем случае, получаем, что $\operatorname{Ext}^{q}\left(E^{\prime \prime}, \mathscr{O}(-1,-1)\right)=0$ для всех $q$.

Из применения функтора $\operatorname{Ext}^{\cdot}(\mathscr{O}, \cdot)$ к последовательности (5) следует, что групшы $\operatorname{Ext}^{q}\left(\mathscr{O}, E^{\prime \prime}\right)$ тривиальны для всех $q$, т.е. $E^{\prime \prime} \in\langle\mathscr{O}(-1,0), \mathscr{O}(0,-1)\rangle_{\text {cat }}$.

Ввиду полной ортогональности пучков $\mathscr{O}(-1,0)$ и $\mathscr{O}(0,-1)$ любое расслоение, принадлежащее категории, ими порожденной, представляется в виде прямой суммы этих пучков. В частности, $E^{\prime \prime}=U_{1} \otimes \mathscr{O}(-1,0) \oplus U_{2} \otimes \mathscr{O}(0,-1)$ для некоторых векторных пространств $U_{1}$ и $U_{2}$. Для вычисления этих пространств к последовательности (5) применим функтор $\operatorname{Ext}^{\cdot}\left(L_{i}, \cdot\right)$, где $L_{1}=\mathscr{O}(1,0), L_{2}=\mathscr{O}(0,1)$. При этом мы получим изоморфизмы

$$
\operatorname{Ext}^{q}\left(L_{i}, E^{\prime}\right) \cong \operatorname{Ext}^{q}\left(L_{i}, E^{\prime \prime}\right) \cong \begin{cases}U_{i} & \text { при } q=1 \\ 0 & \text { при } q \neq 1\end{cases}
$$

Если теперь применим функтор $\operatorname{Ext}\left(L_{i}, \cdot\right), i=1,2$, к последовательности (4), то заметим, что $\operatorname{Ext}^{q}\left(L_{i}, E^{\prime}\right) \cong \operatorname{Ext}^{q}\left(L_{i}, E\right)$.

Осталось собрать последовательности (4) и (5) в одну монаду. Это можно сделать с помощью следующей коммутативной диаграммы:

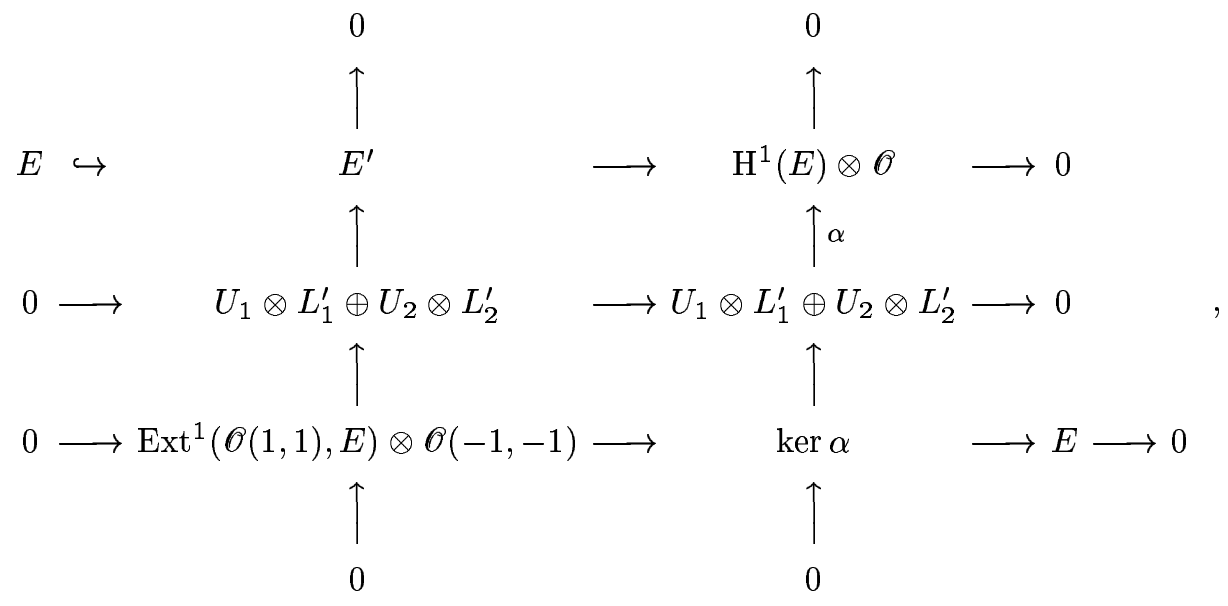

в которой $L_{1}^{\prime}=\mathscr{O}(-1,0), L_{2}^{\prime}=\mathscr{O}(0,-1)$. Предложение доказано. 
ЗАмЕчАнИЕ 1. 1. Предложение 1 справедливо для любого полустабильного пучка $X$ с наклоном $\mu(X) \in(-2,0)$. (Здесь и далее наклоном пучка $X$ ранга $r$ с первьм классом Черна $c_{1}=(a, b)$ называется $\mu(X)=(a+b) / r=\operatorname{deg} x / r$.

2. Монада (2) получается из спектральной последовательности Бейлинсона-Городенцева, построенной по правому двойственному набору к исключительному набору $(\mathscr{O}, \mathscr{O}(1,0), \mathscr{O}(0,1), \mathscr{O}(1,1))[7]$. Поэтому она обладает функториальными свойствами, т.е. морфизм пучков, удовлетворяюших условиям предложения 1 , индуцирует морфизм монад.

ЛЕмма 1. Пучки $X$ и $X^{\prime}$, удовлетворяющие условиям предложсения 1 , изоморфны тогда и только тогда, когда изоморфны их монады.

ДокАЗАТЕЛЬСтво. Очевидно, что изоморфные монады задают изоморфные пучки. С другой стороны, согласно предыдушему замечанию изоморфизм $\varphi: X \rightarrow X^{\prime}$ индуцирует морфизм монад:

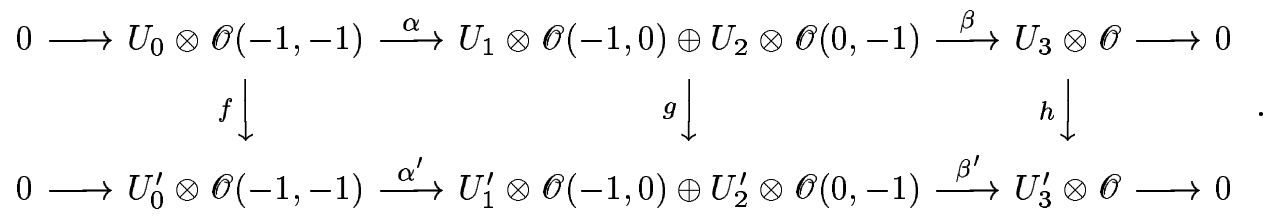

Эта диаграмма разбивается на две:

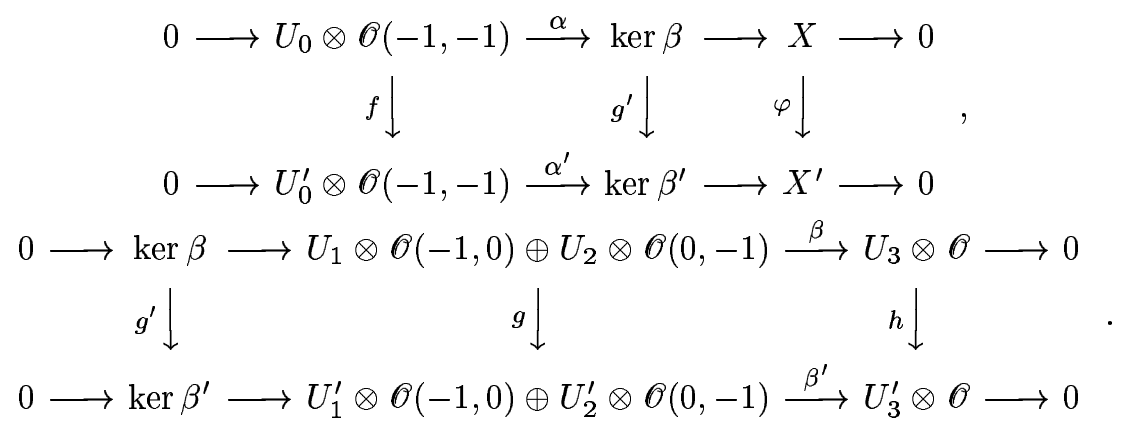

Поскольку $\varphi$ - изоморфизм, согласно лемме о змее из диаграммы (6) следует, что $\operatorname{ker} f \cong \operatorname{ker} g^{\prime}$ и coker $f \cong \operatorname{coker} g^{\prime}$. Линейные расслоения просты. Поэтому $\operatorname{ker} f=$ $V_{0} \otimes \mathscr{O}(-1,-1)$ и с coker $f=W_{0} \otimes \mathscr{O}(-1,-1) ; \operatorname{ker} h=V_{3} \otimes \mathscr{O}$ и coker $h=W_{3} \otimes \mathscr{O}$. Кроме того, из полной ортогональности расслоений $\mathscr{O}(-1,0)$ и $\mathscr{O}(0,-1)$ следует, что

$\operatorname{ker} g=V_{1} \otimes \mathscr{O}(-1,0) \oplus V_{2} \otimes \mathscr{O}(0,-1) \quad$ и $\quad$ coker $g=W_{1} \otimes \mathscr{O}(-1,0) \oplus W_{2} \otimes \mathscr{O}(0,-1)$. Из диаграммы (7) и леммы о змее имеем точную последовательность

$$
\begin{aligned}
& 0 \longrightarrow V_{0} \otimes \mathscr{O}(-1,-1) \longrightarrow V_{1} \otimes \mathscr{O}(-1,0) \oplus V_{2} \otimes \mathscr{O}(0,-1) \longrightarrow V_{3} \otimes \mathscr{O} \\
& \stackrel{\delta}{\longrightarrow} W_{0} \otimes \mathscr{O}(-1,-1) \longrightarrow W_{1} \otimes \mathscr{O}(-1,0) \oplus W_{2} \otimes \mathscr{O}(0,-1) \longrightarrow W_{3} \otimes \mathscr{O} \longrightarrow 0 .
\end{aligned}
$$

Из равенства $\operatorname{Hom}(\mathscr{O}, \mathscr{O}(-1,-1))=0$ следует, что $\delta=0$, и мы получаем две точные тройки, которые возможны лишь в случае тривиальности всех пространств $V_{i}$ и $W_{i}$, так как $\operatorname{Ext}^{1}(\mathscr{O}, \mathscr{O}(-1,-1))=0$. Лемма доказана. 
2. Представления колчана. В работе [4] Кинг изучает многообразие модулей представлений колчана без соотношений. В этом пункте мы сфформулируем результаты Кинга для двух интересующих нас колчанов:

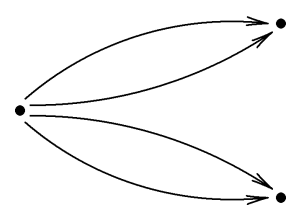

Рис. 1. Колчан $Q_{12}$

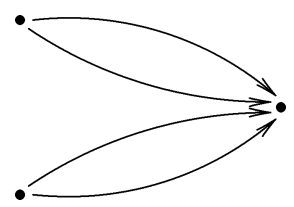

Рис. 2. Колчан $Q_{21}$

ОПРЕДЕЛЕНИЕ. Представлением колчана $Q_{12}$ называется линейное отображение векторных пространств $t: U_{0} \rightarrow U_{1} \otimes L_{1} \oplus U_{2} \otimes L_{2}$, где размерность векторных пространств $L_{i}$ равна 2 . Аналогично, представлением колчана $Q_{21}$ назьвается линейное отображение $t: U_{1} \otimes L_{1} \oplus U_{2} \otimes L_{2} \rightarrow U_{0}$, где $\operatorname{dim} L_{i}=2$.

На пространствах $R_{12}=U_{0}^{*} \otimes\left(U_{1} \otimes L_{1} \oplus U_{2} \otimes L_{2}\right)$ и $R_{21}=R_{12}^{*}=\left(U_{1}^{*} \otimes L_{1}^{*} \oplus U_{2}^{*} \otimes L_{2}^{*}\right) \otimes U_{0}$ действует группа $G=\mathrm{GL}\left(U_{0}\right) \times \mathrm{GL}\left(U_{1}\right) \times \mathrm{GL}\left(U_{2}\right)$ заменой базисов в пространствах $U_{i}$, причем орбиты действия совпадают с классами изоморфных представлений.

Выберем характер группы $G$ :

$$
\chi_{\theta}(g)=\left(\operatorname{det} g_{0}\right)^{\theta_{0}} \cdot\left(\operatorname{det} g_{1}\right)^{\theta_{1}} \cdot\left(\operatorname{det} g_{2}\right)^{\theta_{2}},
$$

где $\left(g_{0}, g_{1}, g_{2}\right) \in G$, и поднимем действие группы $G$ на прямое произведение $R_{i j} \times \mathbb{C}$ по формуле $g(v, z)=\left(g(v), \chi_{\theta}^{-1}(g) \cdot z\right)$.

ОПРеДЕЛЕНИЕ (Кинг). 1. Точка $v \in R_{i j} \chi_{\theta}$-полустабильна, если существует относительный инвариант веса $\chi_{\theta}^{n} f \in \mathbb{C}\left[R_{i j}\right]^{G, \chi_{\theta}^{n}}$ с $n \geqslant 1$ такой, что $f(v) \neq 0$.

2. Точка $v \in R_{i j} \chi_{\theta}$-стабильна, если она $\chi_{\theta}$-полустабильна, $\operatorname{dim} G \cdot v=\operatorname{dim} G / G_{0}$, где $G_{0}$ - ядро действия, и действие группы $G$ на множестве $\left\{v \in R_{i j} \mid f(v) \neq 0\right\}$ замкнуто.

ЗАМЕЧАнИЕ 2. Относительным инвариантом $f \in \mathbb{C}\left[R_{i j}\right]^{G, \chi}$ веса $\chi$ называется функция $f(v)$ на $R_{i j}$, обладающая свойством $f(g \cdot v)=\chi(g) \cdot f(v)$.

Зафиксируем вектор размерностей $\mathbf{u}=\left(u_{0}, u_{1}, u_{2}\right)$, где $u_{i}=\operatorname{dim} U_{i}$, и выберем характер $\theta=\left(\theta_{0}, \theta_{1}, \theta_{2}\right)$ из тех соображений, что

$$
\sum \theta_{i} u_{i}=0
$$

Это означает, что характер $\chi_{\theta}$ на ядре действия групшы $G$ равен 1.

ОпредЕЛЕниЕ. Линейное отображение $t^{\prime}: U_{0}^{\prime} \rightarrow U_{1}^{\prime} \otimes L_{1} \oplus U_{2}^{\prime} \otimes L_{2}$ называется подпредставлением представления $t$, если $U_{i}^{\prime} \subset U_{i}$ и имеет место коммутативная диаграмма

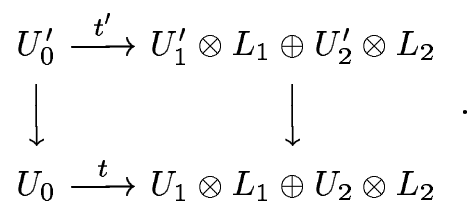

Подпредставление представления $U_{1} \otimes L_{1} \oplus U_{2} \otimes L_{2} \rightarrow U_{0}$ определяется аналогично. 
Теорема 1 (Кинг). Представление $t \in R_{i j} \chi_{\theta}$-стабильно тогда и только тогда, когда для любого его подпредставления $t^{\prime}$ с вектором размерностей $\mathbf{u}^{\prime}$ выполнено условие $\sum \theta_{i} u_{i}^{\prime}>0$ ( $\geqslant 0$ для полустабильного случая).

Теорема 2 (Кинг). Если вектор размерностей и неделим, то фактормногообразие

$$
R_{i j} / G=\operatorname{Proj}\left(\bigoplus_{n \geqslant 0} \mathbb{C}\left[R_{i j}\right]^{G, \chi_{\theta}^{n}}\right)=N_{i j}^{\theta}(\mathbf{u})
$$

является тонким многообразием модулей $\chi_{\theta}$-полустабильных представлений.

При этом на многообразии $N_{12}^{\theta}(\mathbf{u})$ существует универсальное расслоение $\mathscr{U}_{0}^{*} \otimes$ $\left(\mathscr{U}_{1} \otimes L_{1} \oplus \mathscr{U}_{2} \otimes L_{2}\right)$, тавтологическое глобальное сечение которого можно интерпретировать как морфизм пучков на $\mathrm{N}_{12}^{\theta}(\mathbf{u})$

$$
\tau: \mathscr{U}_{0} \rightarrow \mathscr{U}_{1} \otimes L_{1} \oplus \mathscr{U}_{2} \otimes L_{2}
$$

Его слой над каждой точкой $t$ совпадает с представлением $t$.

Аналогично, на многообразии $N_{21}^{\theta}(\mathbf{u})$ существует универсальное расслоение $\left(\mathscr{U}_{1}^{*} \otimes L_{1}^{*} \oplus \mathscr{U}_{2}^{*} \otimes L_{2}^{*}\right) \otimes \mathscr{U}_{0}$ с соответствуюшим глобальным сечением.

ЗАмЕчАниЕ 3 . Так как ядро действия групшы $G=\mathrm{GL}\left(U_{0}\right) \times \mathrm{GL}\left(U_{1}\right) \times \mathrm{GL}\left(U_{2}\right)$ состоит из $\left(\lambda \cdot \mathrm{id}_{U_{0}}, \lambda \cdot \mathrm{id}_{U_{1}}, \lambda \cdot \mathrm{id}_{U_{2}}\right)$ и изоморфно $\mathbb{C}$, то размерность многообразия модулей вычисляется по формуле

$$
\operatorname{dim} N_{i j}^{\theta}(\mathbf{u})=2 u_{0}\left(u_{1}+u_{2}\right)-u_{0}^{2}-u_{1}^{2}-u_{2}^{2}+1 .
$$

Для колчана $Q_{12}$ положим $\theta_{0}=-\left(u_{1}+u_{2}\right), \theta_{1}=u_{0}, \theta_{2}=u_{0}$. Тогда условие (8) будет вьполнено, и неравенство теоремы 1 перепишется в виде

$$
\frac{u_{0}^{\prime}}{u_{1}^{\prime}+u_{2}^{\prime}}<\frac{u_{0}}{u_{1}+u_{2}}
$$

(' $\leqslant$ в полустабильном случае). В связи с этим дробь $u_{0} /\left(u_{1}+u_{2}\right)$ обозначим через $\nu_{12}(t)$ и назовем наклоном представления $t$.

Аналогично, выбор характера $\theta_{0}=u_{1}+u_{2}, \theta_{1}=-u_{0}, \theta_{2}=-u_{0}$ для колчана $Q_{21}$ определяет наклон его представления по формуле $\nu_{21}(t)=\left(u_{1}+u_{2}\right) / u_{0}$.

Тем самым, представление $t$ колчана $Q_{i j}$ является $\chi_{\theta}$-стабильньм тогда и только тогда, ког да любое его подпредставление имеет меньший наклон.

Всюду далее термин "стабильное представление колчана $Q_{i j}$ " означает $\chi_{\theta}$-стабильное представление относительно характера $\chi_{\theta} \mathrm{c} \theta=\left(-\left(u_{1}+u_{2}\right), u_{0}, u_{0}\right)$ для $Q_{12}$ и $\theta=$ $\left(u_{1}+u_{2},-u_{0},-u_{0}\right)$ для $Q_{21}$. Символ $\theta$ будет опускаться и при обозначении многообразия модулей стабильных представлений.

ЗАмечАниЕ 4. Отображение, ставящее в соответствие представлению $t: U_{0} \rightarrow$ $U_{1} \otimes L_{1} \oplus U_{2} \otimes L_{2}$ двойственное к нему $t^{*}: U_{1}^{*} \otimes L_{1}^{*} \oplus U_{2}^{*} \otimes L_{2}^{*} \rightarrow U_{0}^{*}$, осуществляет изоморфизм многообразий модулей $N_{12}(\mathbf{u})$ и $N_{21}(\mathbf{u})$ с одним и тем же вектором размерностей. 
ДокАЗАТЕЛЬСтво. Для проверки этого факта достаточно указать изоморфизм действия групшы $G$ на пространствах представлений колчанов. На пространстве представлений колчана $Q_{12}$ действует группа $G_{12}=\mathrm{GL}\left(U_{0}\right) \times \mathrm{GL}\left(U_{1}\right) \times \mathrm{GL}\left(U_{2}\right)$, а на представлениях колчана $Q_{21}$ действует группа $G_{21}=\mathrm{GL}\left(U_{0}^{*}\right) \times \operatorname{GL}\left(U_{1}^{*}\right) \times \operatorname{GL}\left(U_{2}^{*}\right)$.

При отождествлении этих групп посредством изоморфизма

$$
\left(g_{0}, g_{1}, g_{2}\right) \mapsto\left(\left(g_{0}^{*}\right)^{-1},\left(g_{1}^{*}\right)^{-1},\left(g_{2}^{*}\right)^{-1}\right)
$$

мы получим согласование действий. Более того, при таком изоморфизме отождествляются и выбранные характеры

$$
\left(-\left(u_{1}+u_{2}\right), u_{0}, u_{0}\right) \mapsto\left(u_{1}+u_{2},-u_{0},-u_{0}\right) .
$$

Поэтому стабильность представления колчанов сохраняется при взятии двойственного.

3. Многообразие модулей расслоений. В этом пункте мы построим тонкоемногообразие модулей стабильных пучков $E$ на квадрике с дискретными инвариантами і), ii) (см. введение), если существует хотя бы один такой стабильньй пучок, и покажем, что все такие пучки локально свободны. Далее символом $\mathscr{E}$ мы обозначаем множество стабильных пучков с дискретными инвариантами і).

Лемма 2. Монада (2) для пучков $E \in \mathscr{E}$ имеет вид

$$
0 \longrightarrow U_{0} \otimes \mathscr{O}(-1,-1) \stackrel{\alpha}{\longrightarrow} U_{1} \otimes \mathscr{O}(-1,0) \oplus U_{2} \otimes \mathscr{O}(0,-1) \longrightarrow 0,
$$

т.е. пучок Е включается в точную последовательность

$$
0 \longrightarrow U_{0} \otimes \mathscr{O}(-1,-1) \stackrel{\alpha}{\longrightarrow} U_{1} \otimes \mathscr{O}(-1,0) \oplus U_{2} \otimes \mathscr{O}(0,-1) \longrightarrow E \longrightarrow 0,
$$

əде $U_{0}=\operatorname{Ext}^{1}(\mathscr{O}(1,1), E), U_{1}=\operatorname{Ext}^{1}(\mathscr{O}(1,0), E), U_{2}=\operatorname{Ext}^{1}(\mathscr{O}(0,1), E)$. При этом $\operatorname{dim} U_{0}=r+a+b, \operatorname{dim} U_{1}=r+b, \operatorname{dim} U_{2}=r+a$.

ДокАЗАТЕльство. Наклон пучка $E$ равен $\mu(E)=(a+b) / r$ и лежит в интервале $(-2,0)$. Поэтому пучок $E$ удовлетворяет предложению 1 . С другой стороны, по формуле Римана-Роха

$$
\chi(\mathscr{O}, E)=r\left(1+\frac{a+b}{r}+\frac{a b-(r+a+b+a b)}{r}\right)=0 .
$$

Следовательно, пространство $U_{3}$ из монады для $E$ тривиально. Подсчет размерностей пространств $U_{i}$ проводится аналогично.

Таким образом, каждому пучку $E \in \mathscr{E}$ можно сопоставить мономорфизм пучков

$$
\alpha: U_{0} \otimes \mathscr{O}(-1,-1) \rightarrow U_{1} \otimes \mathscr{O}(-1,0) \oplus U_{2} \otimes \mathscr{O}(0,-1)
$$

т.е. элемент линейного пространства

$$
U_{0}^{*} \otimes\left(U_{1} \otimes \operatorname{Hom}(\mathscr{O}(-1,-1), \mathscr{O}(-1,0)) \oplus U_{2} \otimes \operatorname{Hom}(\mathscr{O}(-1,-1), \mathscr{O}(0,-1))\right) .
$$

Введем обозначения

$$
H_{01}=\operatorname{Hom}(\mathscr{O}(-1,-1), \mathscr{O}(-1,0)), \quad H_{02}=\operatorname{Hom}(\mathscr{O}(-1,-1), \mathscr{O}(0,-1)) .
$$

Как известно, $\operatorname{dim} H_{0 i}=2$ и мономорфизм пучков $\alpha$ однозначно соответствует представлению колчана $t_{\alpha}$ из п. 2 ; и наоборот, каждому представлению колчана $t$ однозначно соответствует морфизм (но необязательно мономорфизм) пучков $\alpha_{t}$. 
Лемма 3. Пусть $t: \mathbb{C}^{u_{0}} \rightarrow \mathbb{C}^{u_{1}} \otimes H_{01} \oplus \mathbb{C}^{u_{2}} \otimes H_{02}-$ представление колчана $u$

$$
\alpha_{t}: \mathbb{C}^{u_{0}} \otimes \mathscr{O}(-1,-1) \rightarrow \mathbb{C}^{u_{1}} \otimes \mathscr{O}(-1,0) \oplus \mathbb{C}^{u_{2}} \otimes \mathscr{O}(-1,0)
$$

- соответствуюший ему морфизм пучков. Если $t$ полустабилен и $\nu_{12}(t)<1 / 2$, то $\alpha_{t}$ - вложение расслоений.

ДокАзАтЕЛьСтво. Представление $t$ задается матрицей $F$ размера $u_{0} \times\left(u_{1}+u_{2}\right) \mathrm{c}$ элементами $\varphi_{i j} \in H_{01} \cup H_{02}$. Рассмотрим ограничение морфизма $\alpha_{t}$ на слой в точке $x$

$$
\alpha_{t x}: \mathbb{C}^{u_{0}} \otimes \mathscr{O}(-1,-1)_{x} \rightarrow \mathbb{C}^{u_{1}} \otimes \mathscr{O}(-1,0)_{x} \oplus \mathbb{C}^{u_{2}} \otimes \mathscr{O}(-1,0)_{x}
$$

Морфизм векторных пространств $\alpha_{t x}$ задается матрицей $F_{x}$, которая получается из матрицы $F$, если вместо $\varphi_{i j}$ поставить их ограничение на слой $\left(\varphi_{i j}\right)_{x}$. Если $\alpha_{t x}$ имеет ненулевое ядро, то, выбрав соответствующий базис в пространстве $\mathbb{C}^{u_{0}}$, мы можем считать, что $\alpha_{t x}\left(e_{1}\right)=0$, т.е. первый базисный вектор пространства $\mathbb{C}^{u_{0}}$ при этом переходит в нуль. Заметим, что если морфизмы $\varphi_{i 1} \in H_{01}$ обрашаются в нуль в одной точке, то они пропорциональны. Аналогичное утверждение справедливо и для элементов $H_{02}$. Тем самым, мы получили, что образ первого базисного вектора при отображении $t$ лежит в двумерном векторном пространстве $\langle\varphi\rangle \oplus\langle\psi\rangle \subset H_{01} \oplus H_{02}$, т.е. $t$ имеет подпредставление $t^{\prime}$ с вектором размерностей $(1,1,1)$. Из сравнения наклонов $\nu(t)<\nu\left(t^{\prime}\right)=1 / 2$ и полустабильности $t$ следует, что $\alpha_{t x}$ не имеет ядра. Следовательно, $\alpha_{t}-$ вложение расслоений.

Для двух пучков $E, F$ без кручения на квадрике положим по определению

$$
d(E, F)=r(E) \operatorname{deg} F-r(F) \operatorname{deg} E=r(E) r(F)(\mu(F)-\mu(E)) .
$$

Для ограниченных комплексов $K^{\cdot}, L^{\cdot}$ пучков примем обозначение

$$
d\left(K^{\cdot}, L^{\cdot}\right)=\sum_{i, j}(-1)^{i+j} d\left(K^{i}, L^{j}\right)
$$

Лемма 4. Пусть $E_{0}, E_{1}, E_{2}$ - расслоения на квадрике такие, что $r\left(E_{1}\right)=$ $r\left(E_{2}\right)=r_{1} u \mu_{0}=\mu\left(E_{0}\right)<\mu\left(E_{1}\right)=\mu\left(E_{2}\right)=\mu_{1}$. Пусть

$$
K^{\prime}: E_{0} \otimes \mathbb{C}^{u_{0}} \rightarrow E_{1} \otimes \mathbb{C}^{u_{1}} \oplus E_{2} \otimes \mathbb{C}^{u_{2}}, \quad L: E_{0} \otimes \mathbb{C}^{u_{0}^{\prime}} \rightarrow E_{1} \otimes \mathbb{C}^{u_{1}^{\prime}} \oplus E_{2} \otimes \mathbb{C}^{u_{2}^{\prime}}
$$

причем $K^{-1}=E_{0} \otimes \mathbb{C}^{u_{0}}$ и $L^{-1}=E_{0} \otimes \mathbb{C}^{u_{0}^{\prime}}$. Тогда

$$
\frac{u_{0}}{u_{1}+u_{2}}>\frac{u_{0}^{\prime}}{u_{1}^{\prime}+u_{2}^{\prime}} \Longleftrightarrow d\left(K^{\cdot}, L^{\cdot}\right)<0 .
$$


ДокАЗАТЕЛЬСТво. По определению функции $d(\cdot, \cdot)$ имеем

$$
d\left(K^{\cdot}, L^{\cdot}\right)=d\left(K^{0}, L^{0}\right)+d\left(K^{-1}, L^{-1}\right)-d\left(K^{0}, L^{-1}\right)-d\left(K^{-1}, L^{0}\right) .
$$

Используя формулу (9) и условие леммы, получаем

$$
\begin{gathered}
d\left(K^{0}, L^{0}\right)=d\left(K^{-1}, L^{-1}\right)=0, \\
d\left(K^{0}, L^{-1}\right)=r_{0} r_{1} u_{0}^{\prime}\left(u_{1}+u_{2}\right)\left(\mu_{0}-\mu_{1}\right), \\
d\left(K^{-1}, L^{0}\right)=r_{0} r_{1} u_{0}\left(u_{1}^{\prime}+u_{2}^{\prime}\right)\left(\mu_{1}-\mu_{0}\right),
\end{gathered}
$$

где $r_{0}=r\left(E_{0}\right)$. Поэтому

$$
d\left(K^{\cdot}, L^{\cdot}\right)=\left(\mu_{1}-\mu_{0}\right) r_{0} r_{1}\left(u_{0}^{\prime}\left(u_{1}+u_{2}\right)-u_{0}\left(u_{1}^{\prime}+u_{2}^{\prime}\right)\right) .
$$

По предположению леммы разность $\mu_{1}-\mu_{0}$ положительна, и знак $d\left(K^{*}, L^{*}\right)$ совпадает со знаком $u_{0}^{\prime}\left(u_{1}+u_{2}\right)-u_{0}\left(u_{1}^{\prime}+u_{2}^{\prime}\right)$. Лемма доказана.

ЗАмЕчАниЕ 5. Предположим, что морфизмы в комплексах из леммы 4 являются вложениями и $F_{K}, F_{L}-$ их коядра. Тогда $d\left(K^{*}, L^{*}\right)=d\left(F_{K}, F_{L}\right)$.

ЛЕмма 5. Пусть морфизм $\alpha$ из точной тройки

$$
0 \longrightarrow U_{0} \otimes \mathscr{O}(-1,-1) \stackrel{\alpha}{\longrightarrow} U_{1} \otimes \mathscr{O}(-1,0) \oplus U_{2} \otimes \mathscr{O}(0,-1) \longrightarrow F \longrightarrow 0
$$

соответствует представлению колчана $t: U_{0} \rightarrow U_{1} \otimes H_{01} \oplus U_{2} \otimes H_{02}$. Тогда из полустабильности пучка $F$ вытекает полустабильность представления $t$.

ДокАЗАТЕЛЬСтво. Обозначим для сокращения записи формул

$$
E_{0}=\mathscr{O}(-1,-1), \quad E_{1}=\mathscr{O}(-1,0), \quad E_{2}=\mathscr{O}(0,-1) .
$$

Предположим, что существует дестабилизирующее подпредставление

$$
t^{\prime}: U_{0}^{\prime} \rightarrow U_{1}^{\prime} \otimes H_{01} \oplus U_{2}^{\prime} \otimes H_{02},
$$

т.е. если $u_{i}^{\prime}=\operatorname{dim} U_{i}^{\prime}$ и $u_{i}=\operatorname{dim} U_{i}$, то

$$
\frac{u_{0}}{u_{1}+u_{2}}<\frac{u_{0}^{\prime}}{u_{1}^{\prime}+u_{2}^{\prime}}
$$

Обозначим через $\alpha^{\prime}: U_{0}^{\prime} \otimes E_{0} \rightarrow U_{1}^{\prime} \otimes E_{1} \oplus U_{2}^{\prime} \otimes E_{2}$ соответствующее ему отображение пучков. При этом возникает коммутативная диаграмма

0

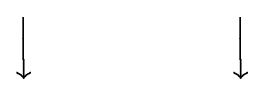

0

$\longrightarrow U_{0}^{\prime} \otimes E_{0} \longrightarrow U_{0} \otimes E_{0} \longrightarrow\left(U_{0} / U_{0}^{\prime}\right) \otimes E_{0} \longrightarrow 0$

$\alpha^{\prime} \downarrow$

0

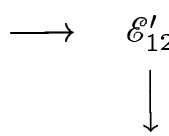

$\operatorname{ker} \alpha^{\prime \prime} \hookrightarrow \quad F^{\prime}$
0
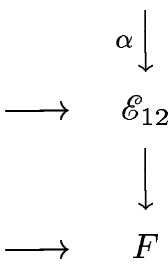
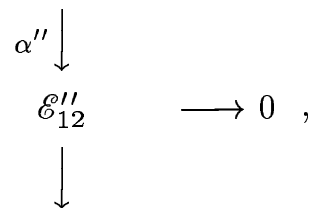

$\longrightarrow \operatorname{coker} \alpha^{\prime \prime} \longrightarrow 0$ 
где $\mathscr{E}_{12}=U_{1} \otimes E_{1} \oplus U_{2} \otimes E_{2}$. Заметим, что мономорфность $\alpha^{\prime}$ следует из леммы о змее.

Если обозначить

$$
K^{\prime}: U_{0} \otimes E_{0} \rightarrow \mathscr{E}_{12}, \quad K^{0}=\mathscr{E}_{12}, \quad L: U_{0}^{\prime} \otimes E_{0} \rightarrow \mathscr{E}_{12}^{\prime}, \quad L^{0}=\mathscr{E}_{12}^{\prime}
$$

то по предположению и лемме 4 справедливо неравенство $d\left(K^{\cdot}, L^{\cdot}\right)>0$. В силу замечания 5 и формулы $(9)$ оно равносильно $\mu(F)<\mu\left(F^{\prime}\right)$.

Средний столбец диаграммы и условие на наклоны расслоений $E_{i}$ влечет неравенство $\mu(F)>\mu\left(E_{0}\right)$. С другой стороны, $\mu\left(\operatorname{ker} \alpha^{\prime \prime}\right) \leqslant \mu\left(E_{0}\right)$, поскольку ker $\alpha^{\prime \prime}-$ подпучок полустабильного расслоения $E_{0} \otimes\left(U_{0} / U_{0}^{\prime}\right)$, наклон которого совпадает с $\mu\left(E_{0}\right)$. Собирая вместе полученные неравенства, имеем

$$
\mu\left(\operatorname{ker} \alpha^{\prime \prime}\right)<\mu\left(F^{\prime}\right)
$$

Рассмотрим последнюю строку диаграммы (10):

$$
0 \longrightarrow \operatorname{ker} \alpha^{\prime \prime} \stackrel{\varphi}{\longrightarrow} F^{\prime} \stackrel{f}{\longrightarrow} F \stackrel{\psi}{\longrightarrow} \operatorname{coker} \alpha^{\prime \prime} \longrightarrow 0 .
$$

Из ее точности следует, что coker $\varphi=\operatorname{ker} \psi$. С другой стороны, по лемме о качелях из неравенства $(11)$ следует $\mu\left(F^{\prime}\right)<\mu(\operatorname{coker} \varphi)$. Поэтому, если $f \neq 0$, пучок coker $\varphi$ дестабилизирует $F$.

Допустим, что $f$ - нулевой морфизм. Тогда $\operatorname{ker} \alpha^{\prime \prime} \cong F^{\prime}$ и $\mu\left(\operatorname{ker} \alpha^{\prime \prime}\right)=\mu\left(F^{\prime}\right)$, что противоречит неравенству (11). Лемма доказана.

ЛЕмма 6. Предположим, что наклон пучка $F$ из точной последовательности

$$
0 \longrightarrow U_{0} \otimes \mathscr{O}(-1,-1) \stackrel{\alpha}{\longrightarrow} U_{1} \otimes \mathscr{O}(-1,0) \oplus U_{2} \otimes \mathscr{O}(0,-1) \longrightarrow F \longrightarrow 0
$$

удовлетворяет условию $-2<\mu(F)<0$. Тогда если пучок $F$ не полустабилен, то существует дестабилизирующий его подпучок $F^{\prime}$, включающийся в коммутативную диаграмму

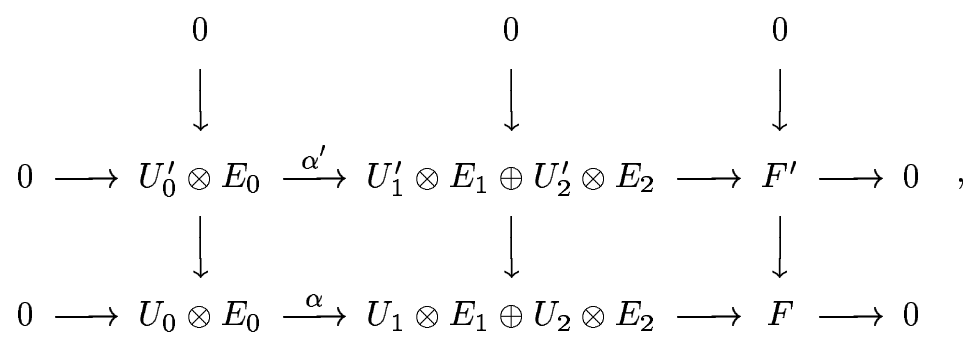

əде, как и раньие, $E_{0}=\mathscr{O}(-1,-1), E_{1}=\mathscr{O}(-1,0), E_{2}=\mathscr{O}(0,-1)$. 
ДокАЗАтЕльство. Рассмотрим фильтрацию Гардера-Нарасимхана пучка $F$ с полустабильными факторами $G_{i}: \operatorname{Gr}(F)=\left(G_{n}, G_{n-1}, \ldots, G_{1}\right)$. Так как наклоны факторов упорядочены $\mu\left(G_{i}\right)<\mu\left(G_{i+1}\right)$ и $\mu\left(G_{1}\right)<\mu(F)<\mu\left(G_{n}\right)$, найдется номер $p$ такой, что $\mu\left(G_{p-1}\right)<\mu(F)<\mu\left(G_{p}\right)$.

Обозначим через $F^{\prime}$ подпучок в $F$ такой, что $\operatorname{Gr}\left(F^{\prime}\right)=\left(G_{n}, \ldots, G_{p}\right)$. Тогда $F^{\prime \prime}=F / F^{\prime}$ имеет фильтрацию $\operatorname{Gr}\left(F^{\prime \prime}\right)=\left(G_{p-1}, \ldots, G_{1}\right)$.

Из точной последовательности (12) следует, что $\operatorname{Ext}^{q}(\mathscr{O}, F)=0$ для всех $q$. Кроме того, из соображений стабильности $\operatorname{Hom}\left(\mathscr{O}, G_{i}\right)=0$ при $i=1,2, \ldots, p-1$. Тем самым,

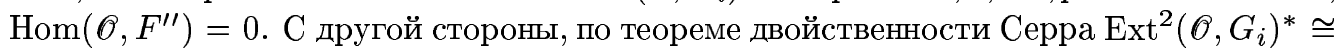
$\operatorname{Hom}\left(G_{i}, \mathscr{O}(-2,-2)\right)$. Поэтому $\operatorname{Ext}^{2}\left(\mathscr{O}, G_{i}\right)=0$ при $i=p, p+1, \ldots, n$ и $\operatorname{Ext}^{2}\left(\mathscr{O}, F^{\prime}\right)=0$.

Теперь из длинной когомологической последовательности, соответствующей точной тройке $0 \rightarrow F^{\prime} \rightarrow F \rightarrow F^{\prime \prime} \rightarrow 0$, вытекает, что $\operatorname{Ext}^{q}\left(\mathscr{O}, F^{\prime}\right)=\operatorname{Ext}^{q}\left(\mathscr{O}, F^{\prime \prime}\right)=0$ для вcex $q$.

Покажем, что

$\operatorname{Ext}^{q}\left(\mathscr{O}(1,0), F^{\prime}\right)=\operatorname{Ext}^{q}\left(\mathscr{O}(0,1), F^{\prime}\right)=\operatorname{Ext}^{q}\left(\mathscr{O}(1,1), F^{\prime}\right)=0$ при $q \neq 1$.

Пусть $\mathscr{L}$ - один из пучков $(\mathscr{O}(1,0), \mathscr{O}(0,1), \mathscr{O}(1,1))$. Из точной последовательности $(12)$ следует, что $\operatorname{Hom}(\mathscr{L}, F)=0$. Поэтому и $\operatorname{Hom}\left(\mathscr{L}, F^{\prime}\right)=0$.

Далее, $\operatorname{Gr}\left(F^{\prime}\right)=\left(G_{n}, \ldots, G_{p}\right)$ и $\mu\left(G_{p}\right)>\mu(F)>-2$. С другой стороны, вьполнено $\mu(\mathscr{L}(-2,-2)) \leqslant-2$, поэтому из соображений стабильности

$$
\operatorname{Ext}^{2}\left(\mathscr{L}, G_{i}\right)^{*} \cong \operatorname{Hom}\left(G_{i}, \mathscr{L}(-2,-2)\right)=0 \text { при } i=p, p+1, \ldots, n,
$$

откуда $\operatorname{Ext}^{2}\left(\mathscr{L}, F^{\prime}\right)=0$. Соотношения (14) доказаны.

Теперь утверждение леммы следует из предложения 1 и замечания 1.

ЛЕммА 7. В предположсения леммы 6 обозначим через

$$
t: U_{0} \rightarrow U_{1} \otimes H_{01} \oplus U_{2} \otimes H_{02}
$$

представление колчана, соответствующее морфизму $\alpha$. Тогда полустабильность представления $t$ влечет полустабильность пучка $F$.

ДокАЗАтЕльство. Пусть $F$ не является полустабильным. Тогда согласно лемме 6 возникает коммутативная диаграмма (13), что позволяет построить подпредставление $t^{\prime}$ представления $t$ :

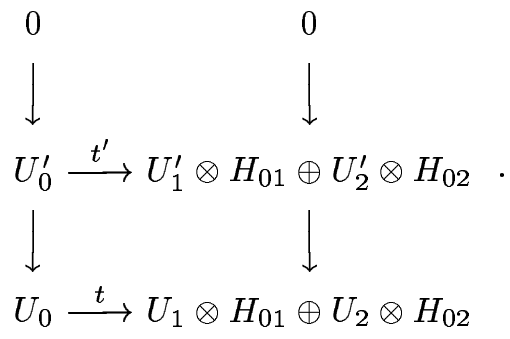

Обозначим через

$$
\begin{array}{cl}
K^{\cdot}: U_{0} \otimes E_{0} \stackrel{\alpha}{\rightarrow} U_{1} \otimes E_{1} \oplus U_{2} \otimes E_{2}, & K^{-1}=U_{0} \otimes E_{0}, \\
L^{\prime}: U_{0}^{\prime} \otimes E_{0} \stackrel{\alpha^{\prime}}{\rightarrow} U_{1}^{\prime} \otimes E_{1} \oplus U_{2}^{\prime} \otimes E_{2}, & L^{-1}=U_{0}^{\prime} \otimes E_{0} .
\end{array}
$$

Так как $d\left(K^{*}, L^{*}\right)=d\left(F, F^{\prime}\right)$ и $F^{\prime}$ дестабилизирует $F$, то $d\left(K^{*}, L^{\cdot}\right)>0$. Это по лемме 4 равносильно тому, что подпредставление $t^{\prime}$ дестабилизирует представление $t$. 
ПРЕДЛОЖЕНИЕ 2. Пусть вектор размерностей $\left(u_{0}, u_{1}, u_{2}\right)$ удовлетворяет условиям:

1) НОД $\left(u_{0}, u_{1}+u_{2}\right)=1$;

2) $-1<\frac{u_{0}}{u_{1}+u_{2}-u_{0}}<1$.

Тогда существует взаимно однозначное соответствие межсду классами изоморфных стабильных представлений

$$
\mathbb{C}^{u_{0}} \rightarrow \mathbb{C}^{u_{1}} \otimes L_{1} \oplus \mathbb{C}^{u_{1}} \otimes L_{1}
$$

колчана $Q_{12}$ и классами изоморфных стабильных пучков $E$ ранга $u_{1}+u_{2}-u_{0} c$ $c_{1}(E)=\left(u_{0}-u_{1}, u_{0}-u_{2}\right)$ и вторым классом Черна $c_{2}=u_{0}+\left(u_{0}-u_{1}\right)\left(u_{0}-u_{2}\right)$. Более того, все такие пучки локально свободны.

ДокАЗАТЕЛЬСтво. Ввиду условия 1) на вектор размерностей любое полустабильное представление является стабильньп.

Перепишем условие 2) на вектор размерностей в виде

$$
-1<\frac{\nu_{12}}{1-\nu_{12}}<1, \quad \text { где } \nu_{12}=\frac{u_{0}}{u_{1}+u_{2}}
$$

Если $\nu_{12}>1$, то левое неравенство равносильно $\nu_{12}-1>\nu_{12}$, что невозможно. Поэтому $\nu_{12}<1$ и правое неравенство равносильно тому, что $\nu_{12}<1 / 2$. С учетом этого неравенства и леммы 3 стабильное представление соответствует вложению расслоений

$$
0 \longrightarrow \mathbb{C}^{u_{0}} \otimes \mathscr{O}(-1,-1) \stackrel{\alpha}{\longrightarrow} \mathbb{C}^{u_{1}} \otimes \mathscr{O}(-1,0) \oplus \mathbb{C}^{u_{2}} \otimes \mathscr{O}(0,-1) .
$$

Его коядро $E$ локально свободно и имеет дискретные инварианты, как в формулировке предложения. Ввиду условия 2) на вектор размерностей наклон расслоения $E$ удовлетворяет неравенству $-2<\mu(E)<0$. Поэтому из леммы 7 следует, что $E$ полустабильно. Покажем, что дробь $\left(2 u_{0}-u_{1}-u_{2}\right) /\left(u_{1}+u_{2}-u_{0}\right)$ несократима, т.е. полустабильность расслоения $E$ влечет его стабильность.

Условие 1) леммы означает, что найдутся целые числа $p$ и $q$ такие, что определитель

$$
\operatorname{det}\left(\begin{array}{cc}
u_{0} & p \\
u_{1}+u_{2} & q
\end{array}\right)=1
$$

При элементарных преобразованиях строк определитель не меняется, поэтому

$$
\operatorname{det}\left(\begin{array}{cc}
u_{0} & p \\
u_{1}+u_{2} & q
\end{array}\right)=\operatorname{det}\left(\begin{array}{cc}
u_{0} & p \\
u_{1}+u_{2}-u_{0} & q-p
\end{array}\right)=\operatorname{det}\left(\begin{array}{cc}
2 u_{0}-u_{1}-u_{2} & 2 p-q \\
u_{1}+u_{2}-u_{0} & q-p
\end{array}\right)=1 .
$$

Пусть теперь $E$ - стабильный пучок с дискретньми инвариантами, удовлетворяющими условиям 1), 2). Тогда по лемме 2 он является коядром мономорфизма

$$
0 \longrightarrow \mathbb{C}^{u_{0}} \otimes \mathscr{O}(-1,-1) \stackrel{\alpha}{\longrightarrow} \mathbb{C}^{u_{1}} \otimes \mathscr{O}(-1,0) \oplus \mathbb{C}^{u_{2}} \otimes \mathscr{O}(0,-1) \longrightarrow E \longrightarrow 0 .
$$

Учитьвая лемму 5, мы видим, что соответствующее представление колчана полустабильно, а значит, и стабильно. Следовательно, $\alpha$ - вложение расслоений и $E$ локально свободен. 
ПРЕДЛОЖЕНИЕ 3. В условиях предложения 2 существует взаимно однозначное соответствие между классами изоморфных стабильных представлений

$$
H_{01}^{*} \otimes \mathbb{C}^{u_{1}} \oplus H_{01}^{*} \otimes \mathbb{C}^{u_{2}} \rightarrow \mathbb{C}^{u_{0}}
$$

колчана $Q_{21}$ и классами изоморфных стабильных пучков $Е$ ранга $u_{1}+u_{2}-u_{0} c$ $c_{1}=\left(-u_{2},-u_{1}\right) u c_{2}=u_{1} u_{2}$.

ДокАЗАТЕЛЬСТво. Наклон пучка $E$ равен $-\left(u_{1}+u_{2}\right) /\left(u_{1}+u_{2}-u_{0}\right)$ и ввиду условия 2$)$ предложения 2 лежит в интервале $(-2,0)$. Из соображений стабильности мы видим, что это влечет условие

$$
\operatorname{Ext}^{q}(\mathscr{L}, E)=0 \text { при } q \neq 1
$$

где $\mathscr{L}$ - один из пучков $\mathscr{O}(-1,-1), \mathscr{O}(-1,0), \mathscr{O}(0,-1), \mathscr{O}$.

Используя формулу Римана-Роха, легко убедиться в том, что $\chi(\mathscr{O}(1,1), E)=0$. Поэтому пучок $E$ включается в точную последовательность

$$
0 \longrightarrow E \longrightarrow U_{1} \otimes \mathscr{O}(0,-1) \oplus U_{2} \otimes \mathscr{O}(-1,0) \stackrel{\alpha}{\longrightarrow} U_{0} \otimes \mathscr{O} \longrightarrow 0
$$

где $U_{1}=\operatorname{Ext}^{1}(\mathscr{O}(0,1), E), U_{2}=\operatorname{Ext}^{1}(\mathscr{O}(1,0), E), U_{0}=\operatorname{Ext}^{1}(\mathscr{O}, E)$ (см. (3)).

Заметим, что пучок $E$, как ядро эпиморфизма расслоений, является локально свободным. Следовательно, применение функтора $\mathscr{E} \mathrm{xt}(\cdot, \mathscr{O})$ к последовательности (15) сохраняет ее точность. Тензорное умножение на обратимьй пучок - также точньй функтор. Поэтому последовательность (15) влечет точную тройку

$$
0 \longrightarrow U_{0}^{*} \otimes \mathscr{O}(-1,-1) \stackrel{\alpha^{\prime}}{\longrightarrow} U_{1}^{*} \otimes \mathscr{O}(-1,0) \oplus U_{2}^{*} \otimes \mathscr{O}(0,-1) \longrightarrow E^{*}(-1,-1) \longrightarrow 0
$$

Поскольку операция '*' и тензорное умножение на обратимьй пучок не влияют на стабильность расслоений, мы видим, что $E^{*}(-1,-1)$ - стабильное расслоение. Значит, представление

$$
t_{\alpha^{\prime}}: U_{0}^{*} \rightarrow U_{1}^{*} \otimes \operatorname{Hom}(\mathscr{O}(-1,-1), \mathscr{O}(-1,0)) \oplus U_{2}^{*} \otimes \operatorname{Hom}(\mathscr{O}(-1,-1), \mathscr{O}(0,-1)),
$$

соответствующее морфизму $\alpha^{\prime}$ стабильно.

Кроме того, представление колчана $Q_{21}$

$$
t_{\alpha}: U_{0} \rightarrow U_{1} \otimes \operatorname{Hom}(\mathscr{O}(0,-1), \mathscr{O}) \oplus U_{2} \otimes \operatorname{Hom}(\mathscr{O}(-1,0), \mathscr{O}),
$$

соответствующее морфизму $\alpha$ из точной тройки $(16)$, двойственно представлению $t_{\alpha^{\prime}}$. Поэтому $t_{\alpha^{\prime}}$ стабильно (замечание 4 ).

С другой стороны, стабильность представления $t_{\alpha}$ влечет стабильность представления $t_{\alpha^{\prime}}$ и расслоения $E^{*}(-1,-1)$, откуда следует стабильность пучка $E$. 
ТЕОремА 3. Пусть иелье числа $r, a, b$ удовлетворяют условиям іi) (см. введение). Предположим, что на квадрике $Q$ существует стабильный пучок ранга $r$ $c c_{1}=(a, b)$ u $c_{2}=r+a+b+a b$. Тогда существует тонкое гладкое компактное многообразие модулей стабильных расслоений с такими дискретными инвариантами, база которого изоморфна $N(r+a+b, r+b, r+a)$.

ДокАЗАТЕЛЬСТво. Из неравенства в іi) и соображений стабильности следует, что $\operatorname{Ext}^{q}\left({ }^{\vee} E_{i}, E\right)=0$ при $q \neq 1$, где упорядоченньй набор $\left({ }^{\vee} E_{3},{ }^{\vee} E_{2},{ }^{\vee} E_{1},{ }^{\vee} E_{0}\right)-$ полный исключительньй набор линейных расслоений на квадрике $(\mathscr{O}, \mathscr{O}(0,1), \mathscr{O}(1,0), \mathscr{O}(1,1))$. Кроме того, размерности групп $\operatorname{Ext}^{1}\left({ }^{\vee} E_{i}, E\right)$ вычисляются по формуле Римана-Роха:

$h^{1}\left({ }^{\vee} E_{0}, E\right)=r+a+b, \quad h^{1}\left({ }^{\vee} E_{1}, E\right)=r+b, \quad h^{1}\left({ }^{\vee} E_{2}, E\right)=r+a, \quad h^{1}\left({ }^{\vee} E_{3}, E\right)=0$.

Легко проверить, что вектор размерностей $(r+a+b, r+b, r+a)$ удовлетворяет условиям 1), 2) предложения 2. Поэтому существует взаимно однозначное соответствие между классами изоморфных стабильных представлений

$$
\mathbb{C}^{r+a+b} \rightarrow \mathbb{C}^{r+b} \otimes H_{01} \oplus \mathbb{C}^{r+a} \otimes H_{02}
$$

и классами изоморфных стабильных пучков $E$ из формулировки теоремы, причем все такие пучки локально свободны. По теореме 2 существует тонкое компактное многообразие модулей $N=N(r+a+b, r+b, r+a)$ таких представлений. Докажем, что оно является тонким многообразием модулей наших стабильных расслоений. Для этого на прямом произведении $Q \times N$ нам необходимо построить пучок, слой которого над $Q \times[E]$ изоморфен $E$, и доказать его универсальность.

Обозначим через $\pi_{Q}$ и $\pi_{N}$ проекции прямого произведения $Q \times N$ на сомножители. На многообразии $N$ существует морфизм универсальных расслоений $\tau: \mathscr{U}_{0} \rightarrow \mathscr{U}_{1} \otimes H_{01} \oplus$ $\mathscr{U}_{2} \otimes H_{02}$, слой которого над точкой $[t]$, изображающей представление $t$, совпадает с ним.

Пусть $\widetilde{\mathscr{U}}_{i}=\pi_{N}^{*} \mathscr{U}_{i}$. Символами $\widetilde{E}_{i}$ обозначим обратные образы линейных расслоений на квадрике при проекции $\pi_{Q}$ :

$$
\widetilde{E}_{i}=\pi_{Q}^{*} E_{i}, \quad E_{0}=\mathscr{O}(-1,-1), \quad E_{1}=\mathscr{O}(-1,0), \quad E_{2}=\mathscr{O}(0,-1) .
$$

Тогда пространства $H_{0 i}$ можно интерпретировать как $\operatorname{Hom}\left(\widetilde{E}_{0}, \widetilde{E}_{i}\right)$, и на прямом произведении $Q \times N$ возникает морфизм расслоений

$$
\widetilde{\alpha}: \widetilde{E}_{0} \otimes \widetilde{\mathscr{U}}_{0} \rightarrow \widetilde{E}_{1} \otimes \widetilde{\mathscr{U}}_{1} \oplus \widetilde{E}_{2} \otimes \widetilde{\mathscr{U}}_{2}
$$

соответствующий морфизму

$$
\widetilde{\tau}: \widetilde{\mathscr{U}}_{0} \rightarrow \widetilde{\mathscr{U}}_{1} \otimes H_{01} \oplus \widetilde{\widetilde{U}_{2}} \otimes H_{02}
$$

Для каждой точки $[t] \in N$ ограничение морфизма $\widetilde{\alpha}$ на $Q \times[t]$ совпадает с морфизмом расслоений на квадрике

$$
\widetilde{\alpha}_{t}: E_{0} \otimes \widetilde{\mathscr{U}}_{0, t} \rightarrow E_{1} \otimes \widetilde{\mathscr{U}}_{1, t} \oplus E_{2} \otimes \widetilde{\mathscr{U}}_{2, t},
$$


соответствующим стабильному представлению. Поэтому $\widetilde{\alpha}_{t}-$ вложение расслоений, а его коядро - стабильное расслоение $E_{t}$. Следовательно, $\widetilde{\alpha}_{t}$ также является вложением расслоений и его коядро $\mathscr{E}$ - нужный нам пучок на прямом произведении.

Рассмотрим произвольное семейство $\mathscr{F}$ стабильных расслоений с нашими дискретными инвариантами с базой $M . \mathrm{K}$ пучку $\mathscr{F}$ на $Q \times M$ сходится спектральная последовательность Бейлинсона-Городенщева с первым членом

$$
\mathbf{E}_{1}^{p, q}=\pi_{M}^{*} \mathscr{E} \mathrm{xt}_{\pi_{M}}^{q}\left(\pi_{Q}^{*}\left({ }^{\vee} E_{3+p}\right), \mathscr{F}\right) \otimes \pi_{Q}^{*} E_{3+p}, \quad q=0,1,2, \quad p=-3,-2,-1,0,
$$

где $\mathscr{E} \mathrm{xt}_{\pi_{M}}^{q}\left(\pi_{Q}^{*}\left({ }^{\vee} E_{3+p}\right), \mathscr{F}\right)=R^{q}\left(\pi_{M}\right)_{*} \mathscr{H} \mathrm{om}\left(\pi_{Q}^{*}\left({ }^{\vee} E_{3+p}\right), \mathscr{F}\right)$. В силу стабильности пучков в семействе $(\mathscr{F}, M)$ и условий на их дискретные инварианты получаем, что

$$
\mathscr{E} \mathrm{xt}_{\pi_{M}}^{q}\left(\pi_{Q}^{*}\left({ }^{\vee} E_{3+p}\right), \mathscr{F}\right)=0 \text { при } q \neq 1
$$

т.е. спектральная последовательность, сходящаяся к пучку $\mathscr{F}$, вырождается в точную тройку. Чтобы ее выписать, положим по определению

$$
H_{3+p}=\mathscr{E x t}_{\pi_{M}}^{1}\left(\pi_{Q}^{*}\left({ }^{\vee} E_{3+p}\right), \mathscr{F}\right)
$$

Тогда

$$
0 \longrightarrow \pi_{M}^{*} H_{0} \otimes \pi_{Q}^{*} E_{0} \stackrel{\widetilde{\beta}}{\longrightarrow} \pi_{M}^{*} H_{1} \otimes \pi_{Q}^{*} E_{1} \oplus \pi_{M}^{*} H_{2} \otimes \pi_{Q}^{*} E_{2} \longrightarrow \mathscr{F} \longrightarrow 0
$$

Легко видеть, что многообразие $M$ с тавтологическим глобальным сечением пучка $H_{0}^{*} \otimes\left(H_{1} \otimes H_{01} \oplus H_{2} \otimes H_{02}\right)$ интерпретируется как семейство представлений колчана с вектором размерностей $(r+a+b, r+b, r+a)$. В силу универсальности семейства $\mathscr{U}_{0}^{*} \otimes\left(\mathscr{U}_{1} \otimes L_{1} \oplus \mathscr{U}_{2} \otimes L_{2}\right)$ над базой $N(r+a+b, r+b, r+a)$ существует морфизм $M \stackrel{\varphi}{\rightarrow} N$ такой, что $H_{i}=\varphi^{*} \mathscr{U}_{i}$. Тогда, очевидно, пучок $\mathscr{F}$ вычисляется как $(\mathrm{id} \times \varphi)^{*}(\mathscr{E})$, где id $\times \varphi: Q \times M \rightarrow Q \times N$. Таким образом, $(M, \mathscr{E})$ является тонким многообразием модулей стабильных пучков. Его компактность очевидна, а гладкость следует из того факта, что касательное пространство к многообразию модулей стабильных пучков на поверхности $\operatorname{Ext}^{1}(E, E)$ имеет постоянную размерность. Теорема доказана.

ТЕОРема 4. Пусть иелье числа $r, a, b$ удовлетворяют условиям іi). Предположим, что на квадрике $Q$ существует стабильный пучок ранга $r$ с $c_{1}=(a, b)$ и $c_{2}=a b$. Тогда существует тонкое гладкое компактное многообразие модулей стабильных расслоений с такими дискретными инвариантами, база которого изоморфна $N(-r-a-b,-a,-b)$.

ДокАЗАТЕЛьСтво. Эта теорема доказьвается аналогично теореме 3 с использованием предложения 3.

4. Многообразие модулей $\mathscr{M}(r,(a, b), k)$. Этот пункт посвящен доказательству основной теоремы. 
ДОКАЗАТЕЛЬСТВо оСНОВНОЙ ТЕОРЕМЫ. Из условия 2) теоремы следует, что наклон пучка $E$ лежит в интервале $(-2,0)$. Поэтому из соображений стабильности этот пучок удовлетворяет условиям предложения 1 , т.е. является когомологией монады (2). Размерности пространств $U_{i}$ легко вычисляются по формуле Римана-Роха:

$\operatorname{dim} U_{0}=-\chi(\mathscr{O}(1,1), E)=k-a b, \quad \operatorname{dim} U_{1}=-\chi(\mathscr{O}(1,0), E)=-k-a b-a$, $\operatorname{dim} U_{2}=-\chi(\mathscr{O}(0,1), E)=-k-a b-b, \quad \operatorname{dim} U_{3}=-\chi(\mathscr{O}, E)=k-r-(a+b)-a b$.

Монада представляется в виде двух точных троек

$$
\begin{gathered}
0 \longrightarrow E^{\prime} \longrightarrow U_{1} \otimes \mathscr{O}(-1,0) \oplus U_{2} \otimes \mathscr{O}(0,-1) \stackrel{\beta}{\rightarrow} U_{3} \otimes \mathscr{O} \longrightarrow 0 \\
0 \longrightarrow U_{0} \otimes \mathscr{O}(-1,-1) \stackrel{\alpha}{\longrightarrow} E^{\prime} \longrightarrow E \longrightarrow 0
\end{gathered}
$$

Из этих последовательностей вычисляются дискретные инварианты пучка $E^{\prime}$ :

$$
r\left(E^{\prime}\right)=r+k-a b, \quad c_{1}\left(E^{\prime}\right)=(a-k+a b, b-k+a b), \quad c_{2}\left(E^{\prime}\right)=(a-k+a b)(b-k+a b) .
$$

Заметим, что $\operatorname{Hom}\left(E^{\prime}, \mathscr{O}(-1,-1)\right)=0$. Действительно, из точной тройки $(17)$ следует, что $E^{\prime}$ принадлежит подкатегории, порожденной тройкой $\langle\mathscr{O}(-1,0), \mathscr{O}(0,-1), \mathscr{O}\rangle_{\text {cat }}$, и $\operatorname{Ext}^{q}\left(E^{\prime}, \mathscr{O}(-1,-1)\right)=0$ для всех $q$. Покажем теперь, что стабильность пучка $E$ равносильна стабильности пучка $E^{\prime}$.

ЛЕмМА 8. Пусть в точной последовательности пучков без кручения на поверхности

$$
0 \longrightarrow V \otimes E^{\prime \prime} \longrightarrow E^{\prime} \longrightarrow E \longrightarrow 0
$$

пучок $E^{\prime \prime}$ локально свободен, стабилен и пространство $\operatorname{Hom}\left(E^{\prime}, E^{\prime \prime}\right)$ тривиально. Предполохим, что наклоны пучков $E^{\prime \prime}$ u $E(\mu(E)=\operatorname{deg} E / r(E))$ удовлетворяют условию

$$
\operatorname{det}\left(\begin{array}{cc}
\operatorname{deg} E^{\prime \prime} & \operatorname{deg} E \\
r\left(E^{\prime \prime}\right) & r(E)
\end{array}\right)=-1
$$

Тогда стабильность пучка Е равносильна стабильности пучка $E^{\prime}$.

ДокАЗАТЕЛЬСТво. Прежде всего заметим, что из условия леммы следует неравенство $\mu\left(E^{\prime \prime}\right)<\mu\left(E^{\prime}\right)<\mu(E)$. Предположим, что пучок $E$ имеет факторпучок $E \rightarrow G \rightarrow 0$ с $\mu(E) \geqslant \mu(G)$. Без ограничения общности можно считать, что пучок $G$ стабилен. Поскольку ранг пучка $G$ строго меньше ранга пучка $E$, условие на определитель влечет неравенство $\mu\left(E^{\prime \prime}\right) \geqslant \mu(G)$. С другой стороны, $\mu\left(E^{\prime}\right)>\mu\left(E^{\prime \prime}\right)$, поэтому $\mu\left(E^{\prime}\right)>\mu(G)$. Тем самьм, $G$ является дестабилизирующим факторпучком у $E^{\prime}$.

Предположим теперь, что пучок $E$ стабилен, а $E^{\prime}$ имеет стабильный факторпучок $G^{\prime}$, удовлетворяющий условию $\mu\left(G^{\prime}\right) \leqslant \mu\left(E^{\prime}\right)$. Как следует из предположений на определитель либо $\mu\left(G^{\prime}\right)<\mu\left(E^{\prime \prime}\right)$, что противоречит стабильности пучка $E^{\prime \prime}$ и наличию ненулевого отображения $\varphi: V \otimes E^{\prime \prime} \rightarrow E^{\prime} \rightarrow G^{\prime}$, либо $\mu\left(G^{\prime}\right)=\mu\left(E^{\prime \prime}\right)$. 
Рассмотрим коммутативную диаграмму

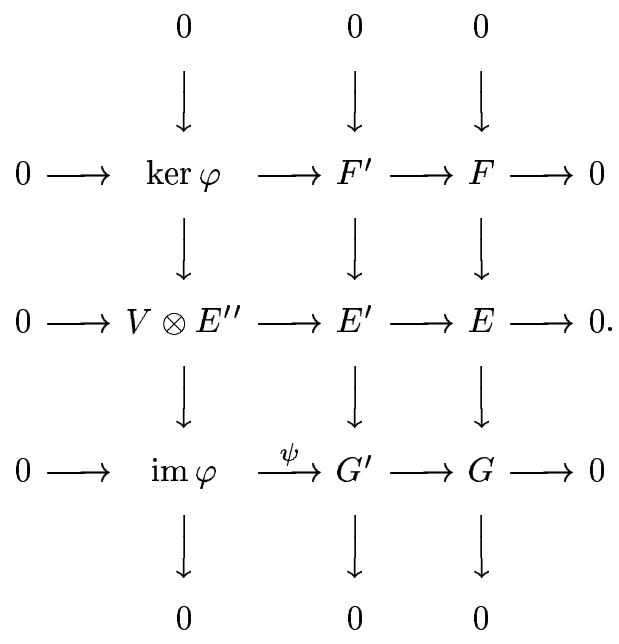

Так как im $\varphi$ - образ морфизма полустабильных пучков равных наклонов, то $\mu(\operatorname{im} \varphi)=$ $\mu\left(E^{\prime \prime}\right)$. Индукцией по числу стабильных факторов в фильтрации Жордана-Гёльдера покажем, что каждый фактор такой фильтрации пучка im $\varphi$ изоморфен $E^{\prime \prime}$. Из левого столбца коммутативной диаграммы (19) следует, что пучок $\operatorname{im} \varphi E^{\prime \prime}$-порожден, т.е. каноническое отображение

$$
\operatorname{can}: \operatorname{Hom}\left(E^{\prime \prime}, \operatorname{im} \varphi\right) \otimes E^{\prime \prime} \rightarrow \operatorname{im} \varphi
$$

является эпиморфизмом, в частности, $\operatorname{Hom}\left(E^{\prime \prime}, \operatorname{im} \varphi\right) \neq 0$. Пусть $\varphi_{1}: E^{\prime \prime} \rightarrow \operatorname{im} \varphi-$ произвольное ненулевое отображение.

Если im $\varphi$ стабилен, то ввиду стабильности $E^{\prime \prime}$ и равенства $\mu(\operatorname{im} \varphi)=\mu\left(E^{\prime \prime}\right)$ отображение $\varphi_{1}-$ изоморфизм вне конечного числа точек [8]. Кроме того, так как пучок $E^{\prime \prime}$ локально свободен и не существует нетривиальных расширений расслоений при помощи пучков с нульмерньм носителем [9], $\varphi_{1}$ - изоморфизм всюду.

Предположим теперь, что im $\varphi$ всего лишь полустабилен, тогда $\varphi_{1}$ - вложение, а к его коядру можно применить предположение индукции.

Теперь обратим внимание на морфизм $\psi$ из диаграммы (19). Он вкладьвает локально свободньй полустабильный пучок $\operatorname{im} \varphi$ в стабильньй пучок $G^{\prime}$, и $\mu\left(G^{\prime}\right)=\mu(\operatorname{im} \varphi)$. Ясно, что такое возможно лишь в случае im $\varphi \cong G^{\prime} \cong E^{\prime \prime}$. Тем самым, мы получили ненулевой элемент из пространства $\operatorname{Hom}\left(E^{\prime}, E^{\prime \prime}\right)$, что противоречит предположению леммы.

Из леммы 8 следует, что каждому классу изоморфных стабильных пучков $E$ из $\mathscr{M}(r,(a, b), k)$ можно однозначно сопоставить класс изоморфньх стабильных пучков $E^{\prime}$ с дискретными инвариантами (18). Простьми вычислениями проверяется, что они удовлетворяют условиям іi). Поэтому согласно теореме 4 каждому классу пучков из $\mathscr{M}(r,(a, b), k)$ сопоставляется точка на многообразии

$$
N=N(k-r-a-b-a b,-k-a b-a,-k-a b-b)
$$


На многообразии $N$ существует универсальное расслоение с сечением, которое интерпретируется как эпиморфизм расслоений

$$
\tau: \mathscr{U}_{1} \otimes H_{13}^{*} \oplus \mathscr{U}_{2} \otimes H_{23}^{*} \rightarrow \mathscr{U}_{3} \rightarrow 0
$$

где $H_{13}=\operatorname{Hom}(\mathscr{O}(-1,0), \mathscr{O}), H_{23}=\operatorname{Hom}(\mathscr{O}(0,-1), \mathscr{O})$. Зафиксируем векторные пространства $H_{0 i}=\operatorname{Hom}\left(\mathscr{O}(-1,-1), E_{i}\right)$, где $E_{1}=\mathscr{O}(-1,0), E_{2}=\mathscr{O}(0,-1), E_{3}=\mathscr{O}$, и определим тензоры $\delta_{i} \in H_{0 i}^{*} \otimes H_{i 3}^{*} \otimes H_{03}$, которые соответствуют спариванию Ионеды $\delta_{i}: H_{0 i} \otimes H_{i 3} \rightarrow H_{03}$. С другой стороны, их можно рассматривать как отображения $\delta_{i}: H_{0 i} \rightarrow H_{i 3}^{*} \otimes H_{03}$. Композицию отображений

$$
\mathscr{U}_{1} \otimes H_{01} \oplus \mathscr{U}_{2} \otimes H_{02} \stackrel{\delta}{\rightarrow} \mathscr{U}_{1} \otimes H_{13}^{*} \otimes H_{03} \oplus \mathscr{U}_{2} \otimes H_{23}^{*} \otimes H_{03} \stackrel{\tau \otimes \mathrm{id}}{\longrightarrow} \mathscr{U}_{3} \otimes H_{03}
$$

обозначим через $\psi$, где $\delta=\mathrm{id} \otimes \delta_{1} \oplus \mathrm{id} \otimes \delta_{2}$.

Пусть $\mathbf{G r}=\operatorname{Gr}\left(\mathscr{U}_{0}, \mathscr{U}_{1} \otimes H_{01} \oplus \mathscr{U}_{2} \otimes H_{02}\right)$ - грассманизация расслоения с подрасслоениями ранга $\operatorname{dim} U_{0}$ и $\mathscr{S}$ - соответствующее универсальное подрасслоение на $\mathbf{G r}$. Обозначим через $\pi_{N}$ проекцию относительного грассманиана на базу $\pi_{N}: \mathbf{G r} \rightarrow N$ и $\widetilde{\mathscr{U}}_{i}=\pi_{N}^{*} \mathscr{U}_{i}$. Тогда на $\mathbf{G r}$ возникает последовательность расслоений

$$
\mathscr{S} \stackrel{\eta}{\longrightarrow} \widetilde{\mathscr{U}}_{1} \otimes H_{01} \oplus \widetilde{\mathscr{U}}_{2} \otimes H_{02} \stackrel{\widetilde{\psi}}{\longrightarrow} \widetilde{\mathscr{U}_{3}} \otimes H_{03}
$$

Обозначим через $\mathscr{Z}$ множество нулей композиции $\widetilde{\psi} \circ \eta$ и покажем, что существует взаимно однозначное соответствие между $\mathscr{M}(r,(a, b), k)$ и $\mathscr{Z}$. Для этого достаточно проверить, что каждому классу изоморфных монад (2) соответствует единственная точка на $\mathscr{Z}$, и наоборот.

Монада (2) определяется парой морфизмов $\alpha$ и $\beta$, которым однозначно соответствуют линейные отображения

$$
t_{\alpha}: U_{0} \rightarrow U_{1} \otimes H_{01} \oplus U_{2} \otimes H_{02} \quad \text { и } \quad t_{\beta}: U_{1} \otimes H_{13}^{*} \oplus U_{2} \otimes H_{23}^{*} \rightarrow U_{3} .
$$

Так как $\alpha$ - мономорфизм, то $t_{\alpha}$, - очевидно, вложение. Следовательно, монада (2), построенная по стабильному пучку $E$, задает точку $z_{E}=\left(t_{\alpha}, t_{\beta}\right)$ в $\mathbf{G r}$. Покажем, что $z_{E} \in \mathscr{Z}$.

Поскольку $\left.\eta\right|_{z_{E}}=t_{\alpha}$, a $\left.\widetilde{\psi}\right|_{z_{E}}=t_{\beta}$, нам достаточно проверить, что композиция

$$
U_{0} \stackrel{t_{\alpha}}{\longrightarrow} U_{1} \otimes H_{01} \oplus U_{2} \otimes H_{02} \stackrel{\delta}{\longrightarrow} U_{1} \otimes H_{13}^{*} \otimes H_{03} \oplus U_{2} \otimes H_{23}^{*} \otimes H_{03} \stackrel{t_{\beta} \otimes \text { id }}{\longrightarrow} U_{3} \otimes H_{03}
$$

нулевая.

Зафиксируем базисы: $\left\{a_{i}\right\}$ в пространстве $U_{0},\left\{b_{j}\right\}$ в пространстве $U_{1},\left\{c_{s}\right\}$ в пространстве $U_{2}$ и $\left\{d_{l}\right\}$ в пространстве $U_{3}$. В этих базисах морфизм $\alpha$ монады задается парой матриц $F^{1}=\left(f_{j i}^{1}\right)$ и $F^{2}=\left(f_{s i}^{2}\right)$, где $f_{j i}^{1} \in H_{01}, f_{s i}^{2} \in H_{02}$. Морфизм $\beta$ также задается парой матриц $G^{1}=\left(g_{l j}^{1}\right)$ и $G^{2}=\left(g_{l s}^{2}\right)$, где $g_{l j}^{1} \in H_{13}, g_{l s}^{2} \in H_{23}$. Тем самьм, композиция $\beta$ о $\alpha$ в матричном виде представляется суммой $G^{1} \cdot F^{1}+G^{2} \cdot F^{2}$, где произведения $g_{l j}^{1} \circ f_{j i}^{1}$ и $g_{l s}^{2} \circ f_{s i}^{2}$ понимаются как композиции отображений. Следовательно, условие $\beta \circ \alpha=0$ равносильно равенству

$$
\sum_{j=1}^{u_{1}} g_{l j}^{1} \circ f_{j i}^{1}+\sum_{s=1}^{u_{2}} g_{l s}^{2} \circ f_{s i}^{2}=0 .
$$


С другой стороны, линейные отображения $t_{\alpha}$ и $t_{\beta}$ в выбранных базисах записьваются теми же матрицами, что и морфизмы монады. Поэтому

$$
t_{\alpha}\left(\sum_{i=1}^{u_{0}} x_{i} \cdot a_{i}\right)=\left(\sum_{i=1}^{u_{0}} x_{i} \sum_{j=1}^{u_{1}} b_{j} \otimes f_{j i}^{1}, \sum_{i=1}^{u_{0}} x_{i} \sum_{s=1}^{u_{2}} c_{s} \otimes f_{s i}^{2}\right) .
$$

Фиксируя базис $\left\{h_{k}\right\}$ в пространстве $H_{03}$, можно записать элемент

$$
\delta \circ t_{\alpha}\left(\sum_{i=1}^{u_{0}} x_{i} \cdot a_{i}\right)
$$

как

$$
\left(\sum_{j=1}^{u_{1}} b_{j} \otimes \sum_{i=1}^{u_{0}} x_{i} \sum_{k=1}^{n} \lambda_{j i}^{1 k} \otimes h_{k}, \sum_{s=1}^{u_{2}} c_{s} \otimes \sum_{i=1}^{u_{0}} x_{i} \sum_{k=1}^{n} \lambda_{s i}^{2 k} \otimes h_{k}\right),
$$

где $\lambda_{j i}^{1 k} \in H_{13}^{*}, \lambda_{s i}^{2 k} \in H_{23}^{*}$ и $\sum_{k=1}^{n} \lambda_{j i}^{1 k} \otimes h_{k}-$ морфизм из $H_{13}$ в $H_{03}$, переводящий элемент $g \in H_{13}$ в композицию $g \circ f_{j i}^{1}$. Сумма

$$
\sum_{k=1}^{n} \lambda_{s i}^{2 k} \otimes h_{k}
$$

определяется аналогично.

Теперь, применяя к (22) отображение $t_{\beta} \otimes \mathrm{id}$, имеем

$$
\sum_{l=1}^{u_{3}} d_{l} \otimes \sum_{i=1}^{u_{0}} x_{i}\left(\sum_{j=1}^{u_{1}} g_{l j}^{1} \circ f_{j i}^{1}+\sum_{s=1}^{u_{2}} g_{l s}^{2} \circ f_{s i}^{2}\right) .
$$

Отсюда и из равенства (21) видно, что точка $z_{E}=\left(t_{\alpha}, t_{\beta}\right)$, соответствующая стабильному пучку $E$, попадает в $\mathscr{Z}$. Более того, эти рассуждения можно обратить и доказать, что если $z_{E}=\left(t_{\alpha}, t_{\beta}\right) \in \mathscr{Z}$, то композиция $\beta \circ \alpha=0$. Итак, мы доказали следующее утверждение:

точки замкнутой подсхемы $\mathscr{Z} \subset \mathbf{G r}$ взаимно однозначно соответствуют классам изоморфных комплексов (2), причем $\beta$ в них всегда эпиморфно.

Проверим, что $\alpha$ в этих комплексах - вложение. Поскольку $\beta$ соответствует стабильному представлению $t_{\beta}$, то его ядро $\operatorname{ker} \beta$ - стабильное расслоение, причем, как легко следует из простых вычислений, наклон $\operatorname{ker} \beta$ удовлетворяет соотношению

$$
\operatorname{det}\left(\begin{array}{cc}
-2 & \operatorname{deg} \operatorname{ker} \beta \\
1 & r(\operatorname{ker} \beta)
\end{array}\right)=-1
$$

Далее, поскольку композиция $\beta$ о $\alpha$ тривиальна, то существует единственное отображение $\alpha^{\prime}: U_{0} \otimes \mathscr{O}(-1,-1) \rightarrow \operatorname{ker} \beta$, ядро которого совпадает с ядром $\alpha$. Пусть $I=\operatorname{im} \alpha$. Тогда из стабильности расслоений $\mathscr{O}(-1,-1)$ и $\operatorname{ker} \beta$ следует

$$
-2 \leqslant \mu(I)<\mu(\operatorname{ker} \beta)
$$


что вместе с соотношением (23) и неравенством $r(I)<r(\operatorname{ker} \beta)$ влечет $\mu(I)=-2$. Отсюда, как и при доказательстве леммы 8 , вытекает $I=U_{0}^{\prime} \otimes \mathscr{O}(-1,-1)$, т.e. $\operatorname{ker} \alpha=\operatorname{ker} \alpha^{\prime}=$ $U_{0}^{\prime \prime} \otimes \mathscr{O}(-1,-1)$. А этот факт противоречит мономорфности $t_{\alpha}$.

Таким образом, мы доказали, что точки замкнутой подсхемы $\mathscr{Z} \subset \mathbf{G r}$ взаимно однозначно соответствуют точкам $\mathscr{M}(r,(a, b), k)$. Теперь осталось ввести структуру тонкого многообразия модулей на $\mathscr{Z}$.

Обозначим ограничение пучков $\widetilde{\mathscr{U}}_{i}$ на $\mathscr{Z}$ символами $\overline{\mathscr{U}}_{i}$ и ограничение $\mathscr{S}$ на $\mathscr{Z}$ через $\overline{\mathscr{S}}$. Тогда последовательность $(20)$ при ограничении на $\mathscr{Z}$ дает комплекс

$$
0 \longrightarrow \overline{\mathscr{S}} \longrightarrow \overline{\mathscr{U}}_{1} \otimes H_{01} \oplus \overline{\mathscr{U}}_{2} \otimes H_{02} \longrightarrow \overline{\mathscr{U}}_{3} \otimes H_{03} \longrightarrow 0
$$

Обозначим проекции прямого произведения $Q \times \mathscr{Z}$ на сомножители $Q$ и $\mathscr{Z}$ через $p_{1}$ и $p_{2}$ соответственно и положим по определению $p_{2}^{*} \overline{\mathscr{U}}_{i}=\mathscr{V}_{i}, p_{2}^{*} \overline{\mathscr{S}}=\mathscr{V}_{0}$. Тогда напрямом произведении $Q \times \mathscr{Z}$ возникает монада

$$
0 \longrightarrow \mathscr{V}_{0} \otimes p_{1}^{*} \mathscr{O}(-1,-1) \longrightarrow \mathscr{V}_{1} \otimes p_{1}^{*} \mathscr{O}(-1,0) \oplus \mathscr{V}_{2} \otimes p_{1}^{*} \mathscr{O}(0,-1) \longrightarrow \mathscr{V}_{3} \otimes p_{1}^{*} \mathscr{O} \longrightarrow 0
$$

с пучком $\mathscr{E}$ в качестве когомологии в среднем члене. Заметим, что по построению ограничение этой последовательности на точку $z_{E} \in \mathscr{Z}$ является монадой, когомология которой изоморфна пучку $E$. Поэтому для доказательства теоремы нам достаточно проверить универсальность семейства $\mathscr{E}$ над базой $\mathscr{Z}$, т.е. доказать, что для любого семейства стабильных пучков $\mathscr{F}$ с дискретными инвариантами из условия основной теоремы над базой $S$ существует отображение $\varphi: S \rightarrow \mathscr{Z}$ такое, что пучок $\mathscr{F}$ на $Q \times S$ изоморфен $(\text { id } \times \varphi)^{*} \mathscr{E}$, где id $\times \varphi: Q \times S \rightarrow Q \times \mathscr{Z}$.

Как уже отмечалось, к пучку $\mathscr{F}$ на $Q \times S$ сходится спектральная последовательность Бейлинсона-Городенцева с первым членом

$$
\mathbf{E}_{1}^{p, q}=\pi_{S}^{*} \mathscr{E} \mathrm{xt}_{\pi_{S}}^{q}\left(\pi_{Q}^{*}\left({ }^{\vee} E_{3+p}\right), \mathscr{F}\right) \otimes \pi_{Q}^{*} E_{3+p}, \quad q=0,1,2, \quad p=-3,-2,-1,0,
$$

где $\pi_{S}$ и $\pi_{Q}-$ проекции прямого произведения $Q \times S$ на сомножители, и

$$
\begin{gathered}
\left(E_{0}, E_{1}, E_{2}, E_{3}\right)=(\mathscr{O}(-1,-1), \mathscr{O}(-1,0), \mathscr{O}(0,-1), \mathscr{O}), \\
\left({ }^{\vee} E_{3},{ }^{\vee} E_{2},{ }^{\vee} E_{1},{ }^{\vee} E_{0}\right)=(\mathscr{O}, \mathscr{O}(0,1), \mathscr{O}(1,0), \mathscr{O}(1,1)) .
\end{gathered}
$$

Как при доказательстве теоремы 3 , мы видим, что эта спектральная последовательность вырождается в монаду

$$
0 \longrightarrow \pi_{S}^{*} F_{0} \otimes \pi_{Q}^{*} E_{0} \stackrel{\alpha}{\longrightarrow} \pi_{S}^{*} F_{1} \otimes \pi_{Q}^{*} E_{1} \oplus \pi_{S}^{*} F_{2} \otimes \pi_{Q}^{*} E_{2} \stackrel{\beta}{\longrightarrow} \pi_{S}^{*} F_{3} \otimes \pi_{Q}^{*} E_{3} \longrightarrow 0
$$

с когомологией $\mathscr{F}$, где $\left.F_{i}=\mathscr{E}_{\mathrm{xt}^{1}}^{1}\left(\pi_{Q}^{*}\left({ }^{\vee} E_{3+p}\right), \mathscr{F}\right)\right)$.

Ограничение этой монады на точку $s_{E} \in S$, соответствующую пучку $E$, является стандартной монадой (2) на квадрике, построенной по $E$. Сопоставляя каждой точке $s_{E} \in S$ представление колчана, соответствующее ограничению $\beta$ на $Q \times s_{E}$, мы получим отображение $\nu: S \rightarrow N$, причем $\nu^{*} \mathscr{U}_{i}=F_{i}, i=0,1,2$, где $\mathscr{U}_{i}$ - универсальные расслоения на $N$. 
Ввиду универсальности относительного грассманиана это отображение поднимается до отображения в $\mathbf{G r} \varphi: S \rightarrow \mathbf{G r}$, причем образ $\nu(S)$ этого отображения содержится в $\mathscr{Z}$, а обратньй образ комплекса (24) при отображении $\varphi$ является монадой

$$
0 \longrightarrow F_{0} \otimes L_{3}^{*} \longrightarrow F_{1} \otimes V_{1}^{*} \oplus F_{2} \otimes V_{2}^{*} \longrightarrow F_{3} \longrightarrow 0 .
$$

Следовательно, $(\mathrm{id} \times \varphi)^{*} \mathscr{E} \cong \mathscr{F}$ и теорема доказана.

В заключение мы покажем, что для любого натурального $k \geqslant 4$ многообразие модулей $\mathscr{M}(3,(-4,-1), k)$ непусто, т.е. существует стабильньй пучок ранга 3 с $c_{1}=(-4,-1)$ и $c_{2}=k$.

Рассмотрим пару обратимых пучков $(\mathscr{O}(-1,0), \mathscr{O}(0,1))$ на квадрике. Очевидно, она является исключительной, т.е.

$$
\operatorname{Ext}^{q}(\mathscr{O}(0,1), \mathscr{O}(-1,0))=0 \quad \forall q, \quad \operatorname{Ext}^{q}(\mathscr{O}(-1,0), \mathscr{O}(0,1))=0 \text { при } q>0 .
$$

Левая перестройка этой пары (см. [2]) определяет исключительное расслоение $E$ :

$$
0 \longrightarrow E \longrightarrow \operatorname{Hom}(\mathscr{O}(-1,0), \mathscr{O}(0,1)) \otimes \mathscr{O}(-1,0) \stackrel{\text { can }}{\longrightarrow} \mathscr{O}(0,1) \longrightarrow 0
$$

Tак как $\operatorname{dim} \operatorname{Hom}(\mathscr{O}(-1,0), \mathscr{O}(0,1))=4$, то $r(E)=3, c_{1}(E)=(-4,-1), c_{2}(E)=4$. Кроме того, исключительные расслоения на квадрике, как и на всех поверхностях Дель Пецщо, стабильны [2].

Наконец, рассмотрим произвольньй эпиморфизм

$$
\pi: E \rightarrow \mathscr{O}_{P_{1}} \oplus \cdots \oplus \mathscr{O}_{P_{d}} \rightarrow 0
$$

где $\mathscr{O}_{P_{i}}-$ небоскребы в точках $P_{i}$. Его ядро - стабильньй пучок ранга 3 с $c_{1}=(-4,-1)$ и $c_{2}=k+d$.

\section{СПИСОК ЦИТИРОВАННОЙ ЛИТЕРАТУРЫ}

[1] Drezet J.-M. Fibrés exceptionnels et variétés de modules de faisceaux semi-stables sur $\mathbb{P}_{2}(\mathbb{C})$ // J. Reine Angew. Math. 1987. V. 380. P. 14-58.

[2] Карпов Б. В. Полустабильные пучки на двумерной квадрике и модули Кронекера // Изв. РАН. Сер. матем. 1992. Т. 56. №1. С. 38-74.

[3] Ellingsrud G., Strömme S. A. Towards the Chow Ring of the Moduli Space for Stable Sheaves on $\mathbb{P}_{2}$ with $c_{1}=1$. Preprint. Oslo: Oslo Univ., 1991.

[4] King A. Moduli of representations of finite-dimensional algebras // Quart. J. Math. Oxford Ser. (2). 1994. V. 45. P. 515-530.

[5] Bondal A. I. Helixes, representation of Quivers and Koszul algebras // Helixes and Vector Bundles. London Math. Soc. Lecture Note Ser. V. 148. Cambridge: Cambridge Univ. Press, 1990. P. $75-95$.

[6] Рудаков А.Н. Исключительные расслоения на квадрике // Изв. АН СССР. Сер. матем. 1988. Т. 52. № 4. C. $788-812$.

[7] Gorodentsev A. L. Exceptional objects and mutations in derived categories // Helixes and Vector Bundles. London Math. Soc. Lecture Note Ser. V. 148. Cambridge: Cambridge Univ. Press, 1990. P. 57-74.

[8] Оконек К., Шнейдер М., Шпиндлер Х. Векторные расслоения на комплексных проективных пространствах. М.: Мир, 1984.

[9] Гриффитс Ф., Харрис Дж. Принципы алгебраической геометрии. М.: Мир, 1982. 